\title{
Cancer immunotherapy beyond immune checkpoint inhibitors
}

\author{
Julian A. Marin-Acevedo ${ }^{1}$, Aixa E. Soyano ${ }^{2}$, Bhagirathbhai Dholaria ${ }^{2,3}$, Keith L. Knutson ${ }^{4}$ and Yanyan Lou ${ }^{2 *}$ (D)
}

\begin{abstract}
Malignant cells have the capacity to rapidly grow exponentially and spread in part by suppressing, evading, and exploiting the host immune system. Immunotherapy is a form of oncologic treatment directed towards enhancing the host immune system against cancer. In recent years, manipulation of immune checkpoints or pathways has emerged as an important and effective form of immunotherapy. Agents that target cytotoxic T lymphocyte-associated molecule-4 (CTLA-4), programmed cell death receptor-1 (PD-1), and programmed cell death ligand-1 (PD-L1) are the most widely studied and recognized. Immunotherapy, however, extends beyond immune checkpoint therapy by using new molecules such as chimeric monoclonal antibodies and antibody drug conjugates that target malignant cells and promote their destruction. Genetically modified T cells expressing chimeric antigen receptors are able to recognize specific antigens on cancer cells and subsequently activate the immune system. Native or genetically modified viruses with oncolytic activity are of great interest as, besides destroying malignant cells, they can increase anti-tumor activity in response to the release of new antigens and danger signals as a result of infection and tumor cell lysis. Vaccines are also being explored, either in the form of autologous or allogenic tumor peptide antigens, genetically modified dendritic cells that express tumor peptides, or even in the use of RNA, DNA, bacteria, or virus as vectors of specific tumor markers. Most of these agents are yet under development, but they promise to be important options to boost the host immune system to control and eliminate malignancy. In this review, we have provided detailed discussion of different forms of immunotherapy agents other than checkpoint-modifying drugs. The specific focus of this manuscript is to include first-in-human phase I and phase I/II clinical trials intended to allow the identification of those drugs that most likely will continue to develop and possibly join the immunotherapeutic arsenal in a near future.
\end{abstract}

Keywords: Immunotherapy, Tumor-directed monoclonal antibodies, Antibody drug conjugates, Chimeric antigen receptor therapy, Oncolytic viruses, Tumor vaccines, Viral gene therapy

\section{Background}

Immunotherapy consists in harnessing the body's own immune system to generate an anti-tumor response, which is often sustained after treatment has finished, suggesting a role of modulation and modification of the immune system $[1,2]$. The most commonly used strategy is the modulation of immune checkpoints, particularly the cytotoxic $\mathrm{T}$ lymphocyte-associated molecule-4 (CTLA-4), programmed cell death receptor-1 (PD-1), or programmed cell death ligand-1 (PD-L1), although new inhibitory (e.g., lymphocyte activation gene/LAG-3, T cell immunoglobulin/TIM3, V-domain Ig suppressor of $\mathrm{T}$ cell activation/VISTA) and

\footnotetext{
* Correspondence: lou.yanyan@mayo.edu

${ }^{2}$ Department of Hematology and Oncology, Mayo Clinic, 4500 San Pablo Road, Jacksonville, FL 32224, USA

Full list of author information is available at the end of the article
}

stimulatory (e.g., inducible co-stimulator/ICOS, OX40, 41BB) pathways have also emerged as targets [3]. Unfortunately, some limitations with immune checkpoint therapy are of concern including a response heterogeneity where some patients achieve a complete response (CR) but others never do. Furthermore, there can be tumor relapse due to alternative immune escape mechanisms, and there is lack of optimal biomarkers to predict response and toxicity. Other major issues have been the emergence of new adverse events and autoimmune-like reactions, and the cost associated with this therapy. Thus, approaches that differ from manipulating immune checkpoints, as alternatives, are under investigation and are the focus of this review. Specifically, we discuss current molecules under phase I and I/II clinical investigation in the area of conjugated monoclonal antibodies (mAbs), chimeric antigen 
receptor (CAR) T cells, oncolytic viruses, vaccines, and other immune-based approaches that are under investigation. Agents in more advanced investigational stages (e.g., phase III) have not been included. Figure 1 summarizes the different strategies that will be discussed, and a summary of these agents is found in Table 1.

\section{Methodology}

We performed a thorough review using the databases PubMed and the American Society of Clinical Oncology (ASCO), both the American Association of Cancer Research (AARC) meeting abstract databases, and ClinicalTrials.gov updated through October 5, 2017. We narrowed our research with the following keywords and $\mathrm{MeSH}$ terms: immunotherapy, tumor directed monoclonal antibodies, antibody drug conjugates, chimeric antigen receptor T-Cells, oncolytic viruses, oncologic vaccines, and adjuvants in immunotherapy. We focused our attention on phase I and phase II clinical trials of new agents in immunotherapy being used with or without other form of immunotherapy. Inclusion criteria included published trials or reported preliminary results during the time of the data collection. Exclusion criteria included phase III or more advanced clinical trials, clinical trials focusing only on immune checkpoint therapy, clinical trials in pediatric population, and non-clinical trials. Finally, we collected 65 phase I and 53 phase II clinical trials for this review.

\section{Tumor-directed monoclonal antibodies}

A range of mAbs directed against tumor-specific antigens are currently under development. These mAbs can bind specific tumor antigens, stay on the surface, and activate antibody/complement-dependent cytotoxicity, or affect downstream signals. Monoclonal antibodies promote tumor killing by different mechanisms including direct cell killing by induction of apoptosis, receptor blockade or agonist activity, delivery of cytotoxic agents, radiation, immune-mediated cell killing, or through specific effects on tumor vasculature and stroma [4]. Of particular interest are the recently developed bispecific antibodies that combine antigen-binding specificities on tumor cells and effector immune cells. Among these, bispecific $\mathrm{T}$ cell engager (BiTE) and dual-affinity re-targeting (DART) are particularly attractive [5-7]. BiTEs recombinantly link the four variable domains of heavy and light chains with a flexible linker peptide allowing to bypass $\mathrm{MHC} /$ peptide

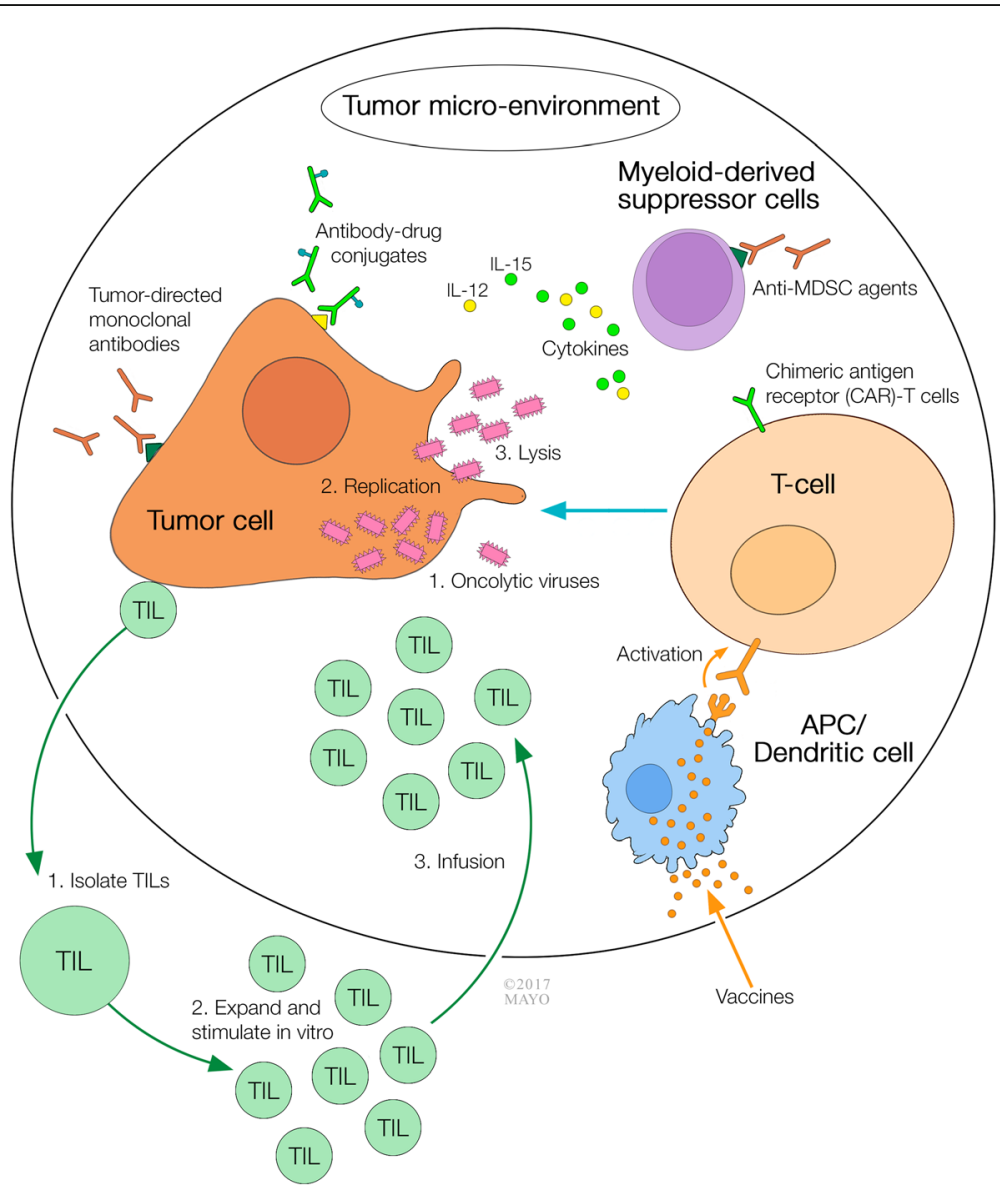

Fig. 1 Multi-modality cancer immunotherapy approaches 


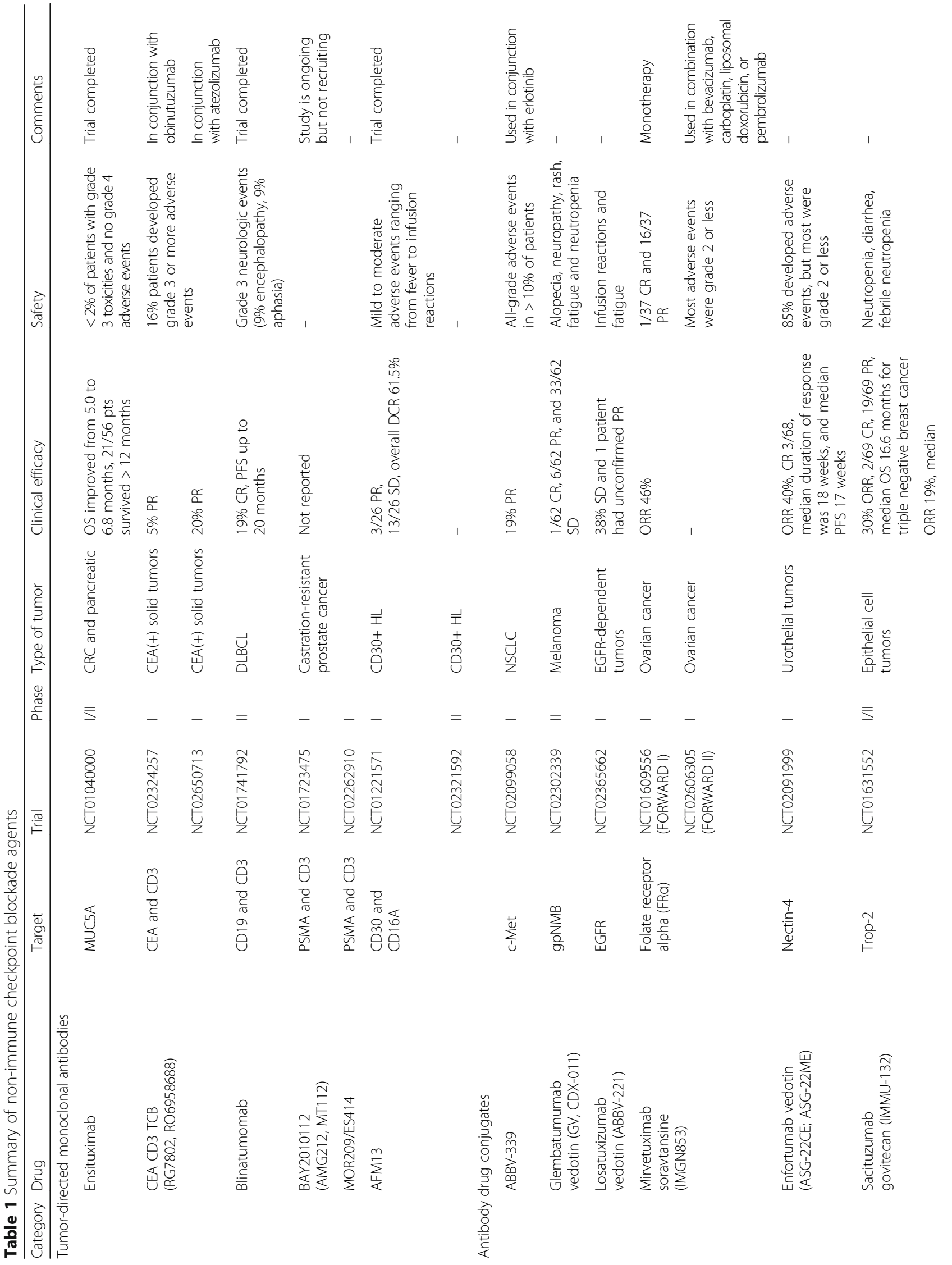


Marin-Acevedo et al. Journal of Hematology \& Oncology (2018) 11:8

Page 4 of 25

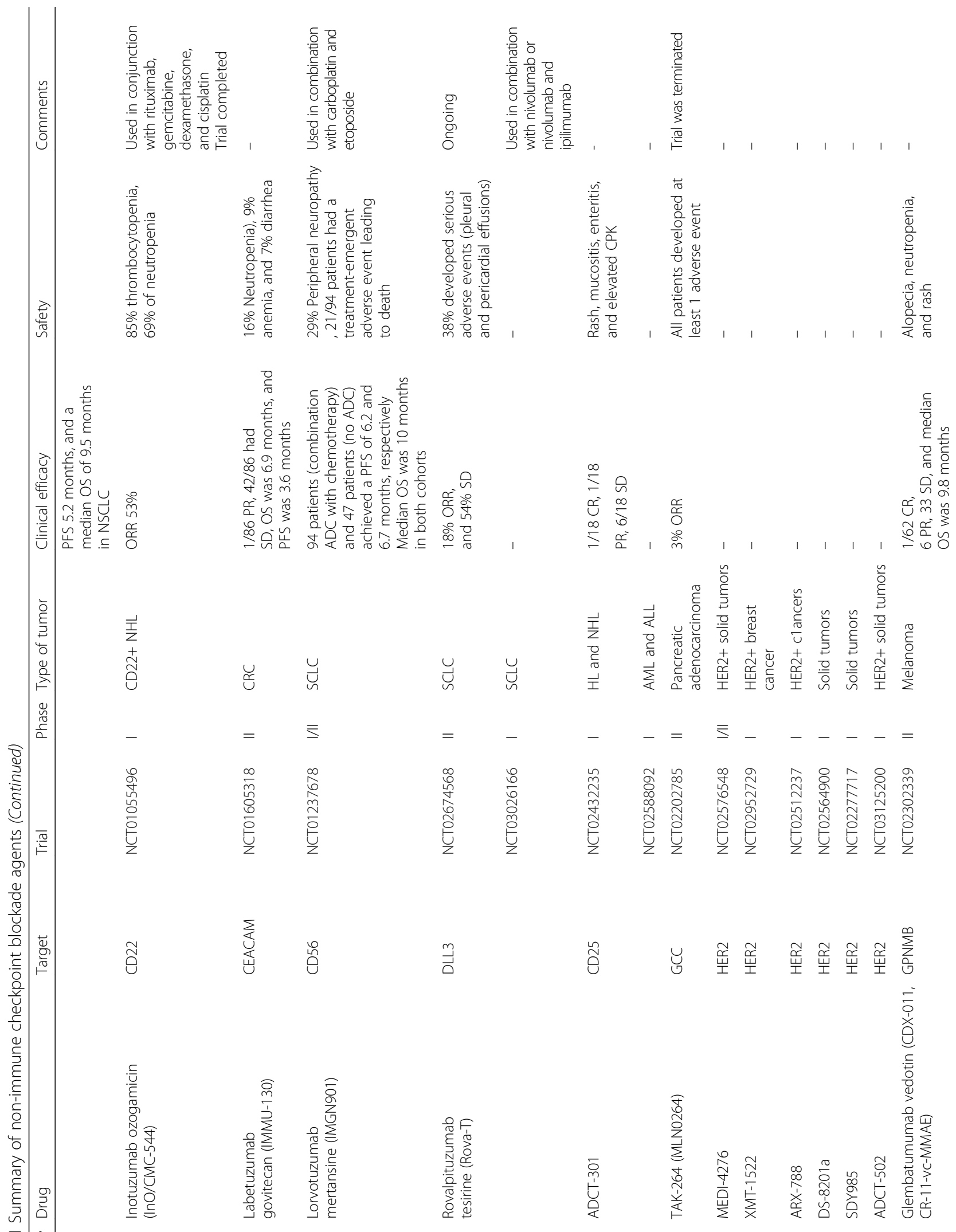




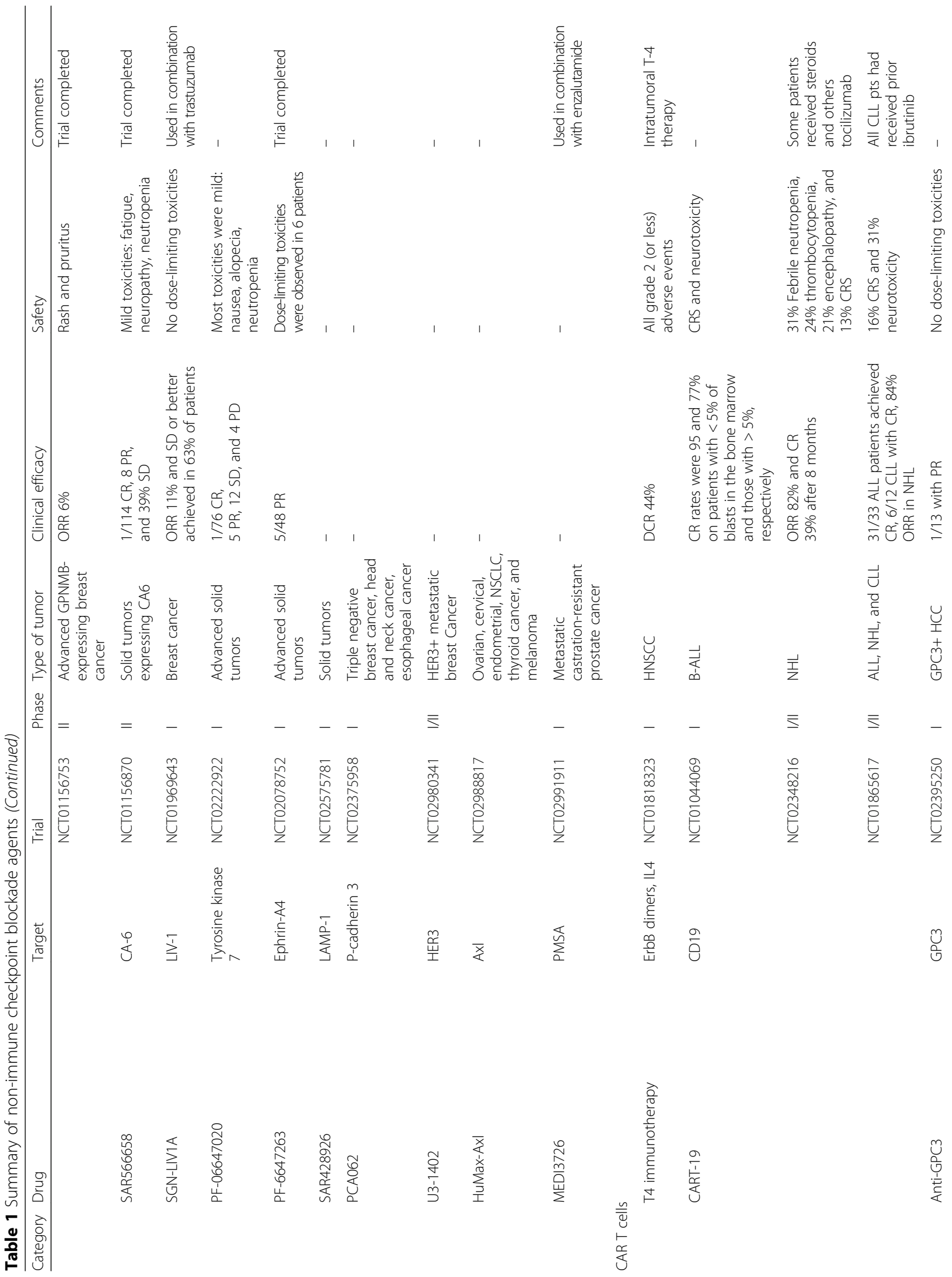




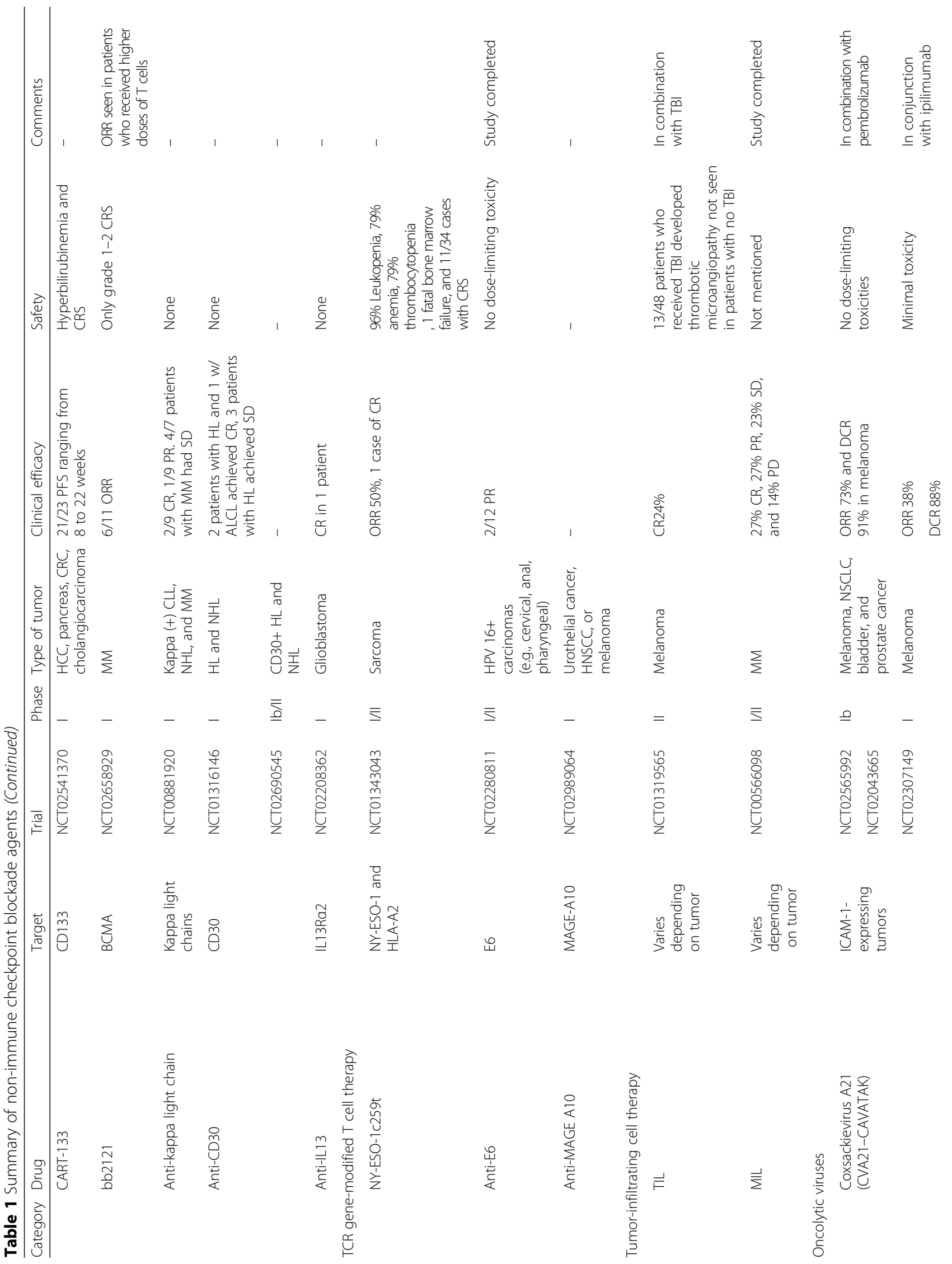




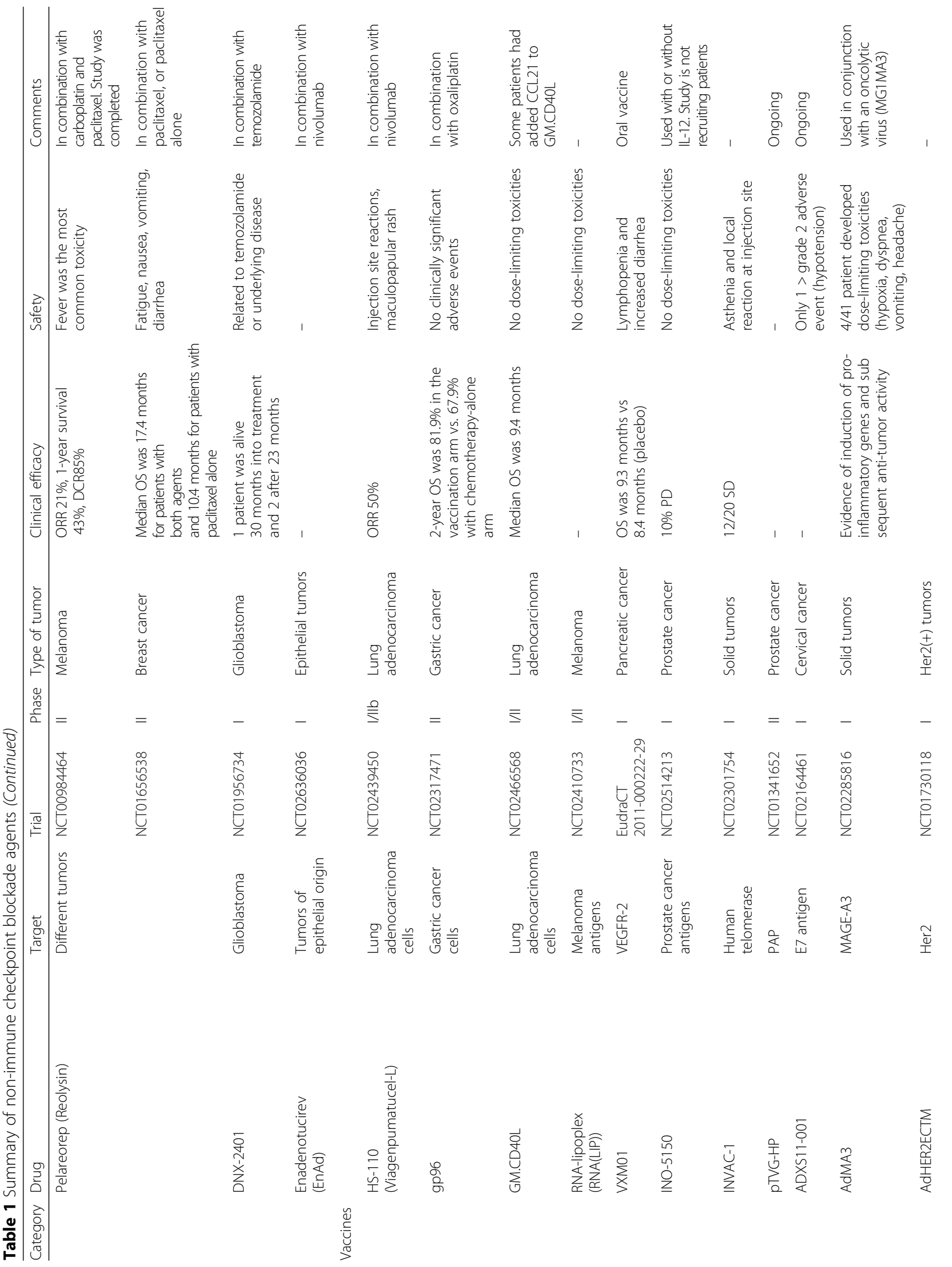




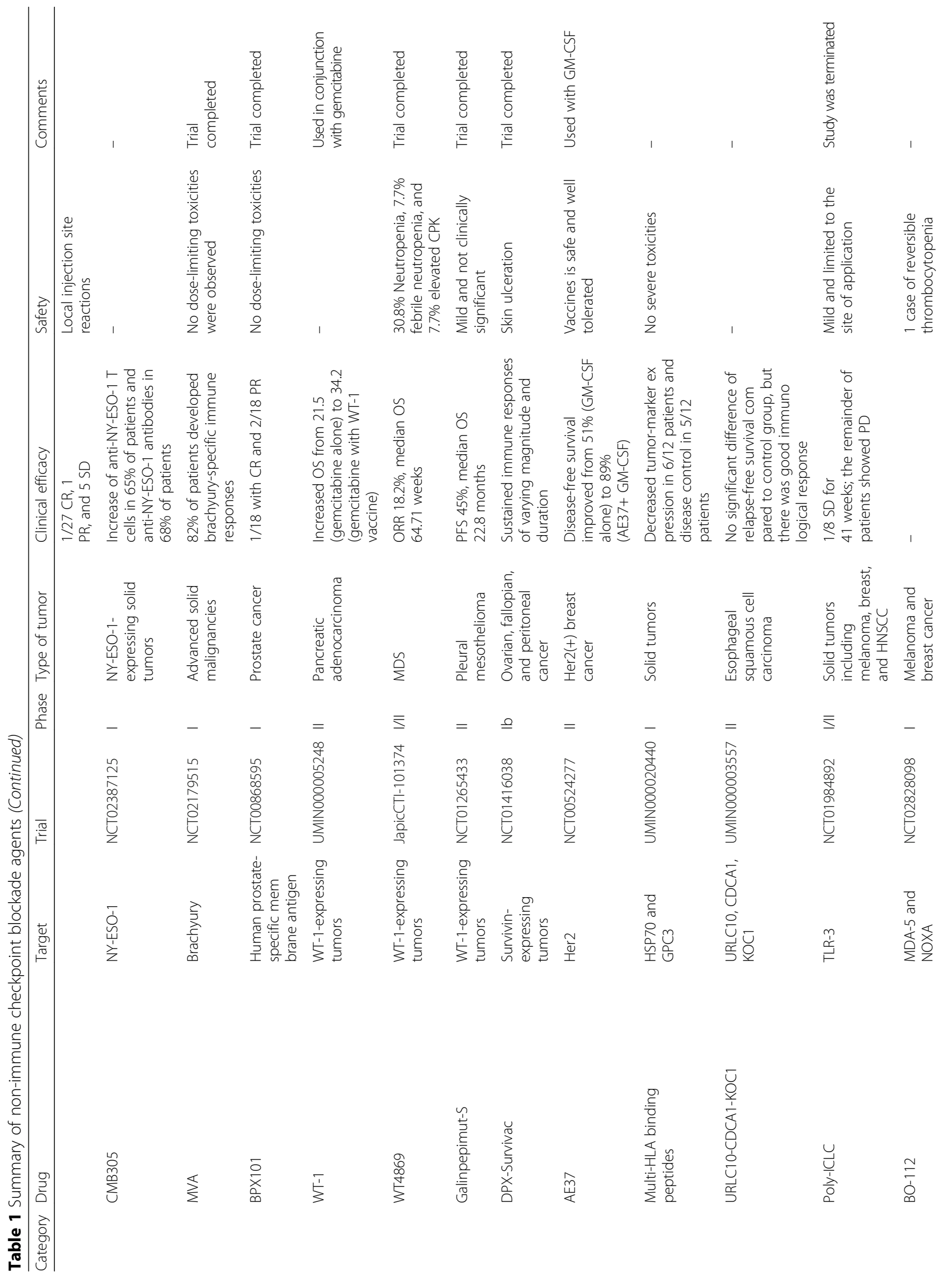




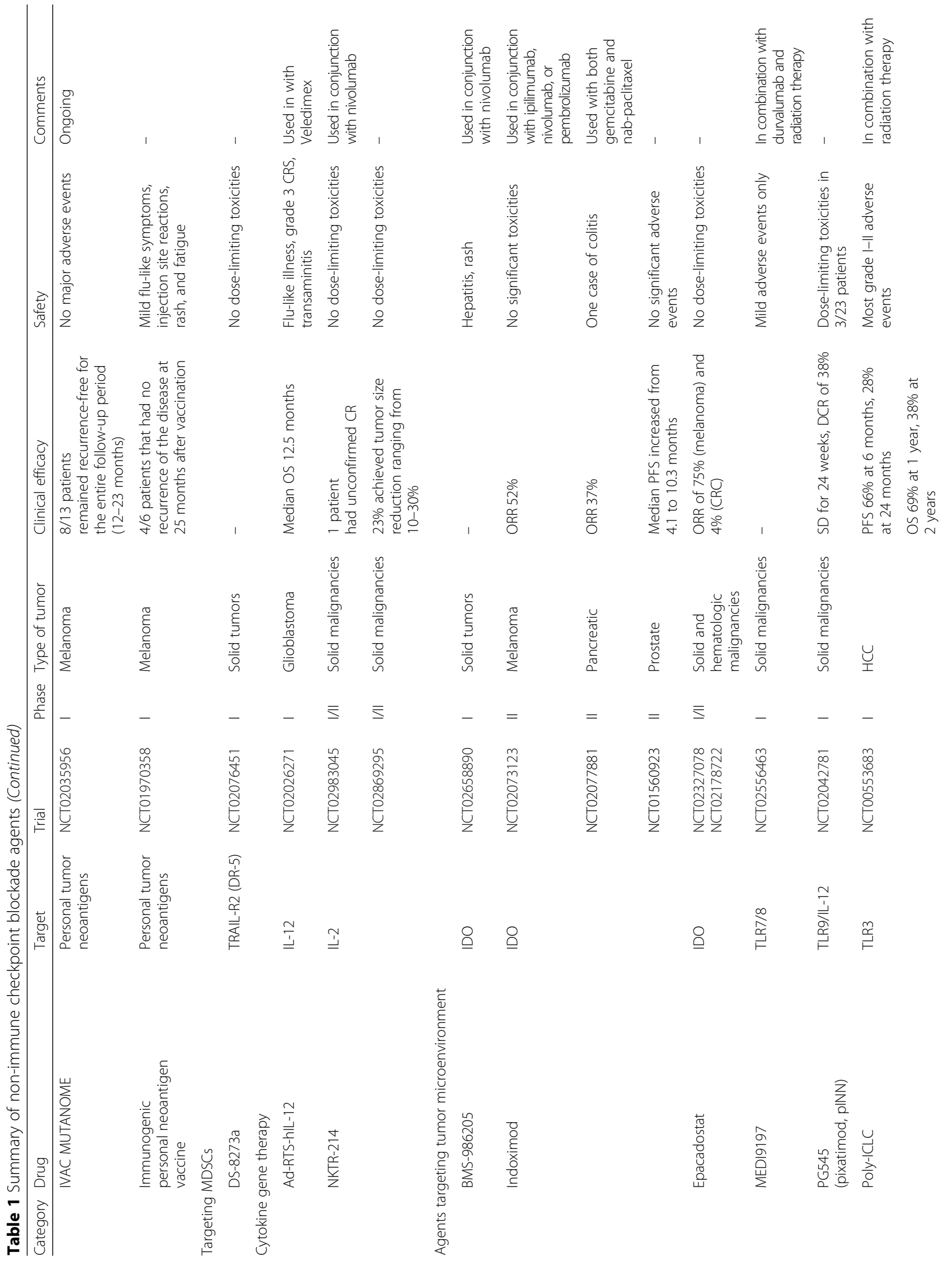




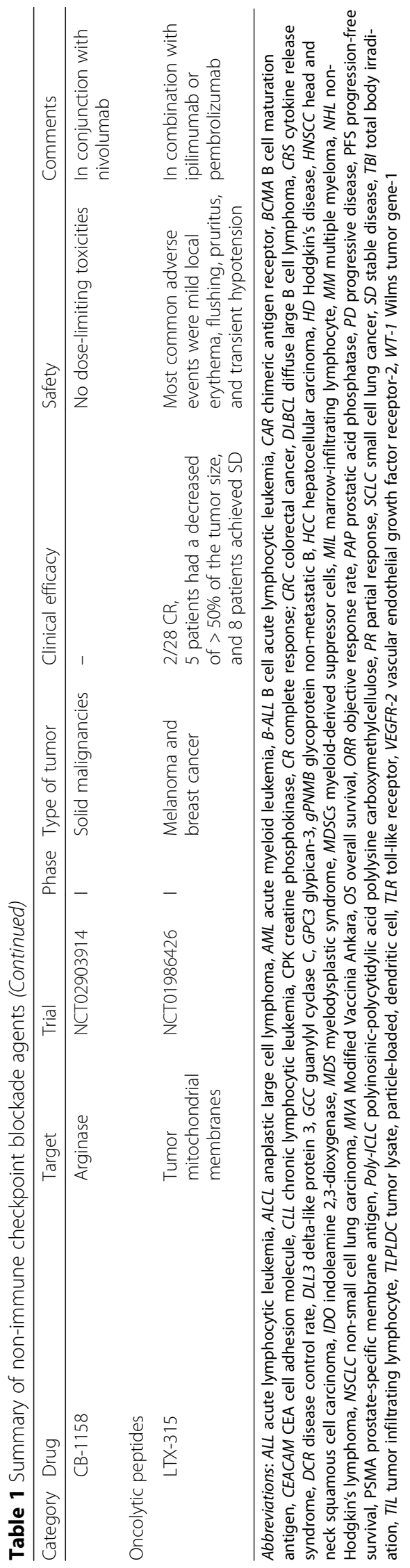


recognition and co-stimulation and also to bring effector cells and target cells close together to form cytolytic synapses. This therapy has revealed impressive clinical activity in relapsed non-Hodgkin's lymphoma, chronic lymphocytic leukemia, and acute lymphoblastic leukemia at doses much lower than those administered in conventional monoclonal antibody therapy. DART consists of a diabody that separates variable domains of heavy and light chains of the two antigen-binding specificities on two separate polypeptide chains stabilized through a C-terminal disulfide bridge which acts as a linker [5]. Compared with BiTE, DART has shown a moderately higher association rate constant for $\mathrm{CD} 3$ and an ability to cross-link T cells and B cells more efficiently $[8,9]$. Ongoing clinical trials will provide more insightful understanding through sideby-side comparison of DART, BiTE, and other bispecific antibody with identical antigen-binding specificities. The quality, stability, and drug distribution of antibodies remain a challenge. Below outlines the ongoing early development of agents within this form of therapy.

Ensituximab (NPC-1C) is a chimeric IgG1 monoclonal antibody that promotes antibody-dependent cellular cytotoxicity after binding its target, a tumor-specific variant of MUC5AC, an antigen that is specifically expressed by colorectal (CRC) and pancreatic cancers. Results from a completed phase I/II clinical trial were recently published and are encouraging [10]. The therapy was well tolerated with $<2 \%$ of patients experiencing grade 3 toxicities and there were no grade 4 adverse events. Furthermore, median overall survival (OS) was significantly longer than historical control: 6.8 vs 5.0 months, 21 out of 56 patients survived $>12$ months.

BiTEs simultaneously target two different antigens and thus target two different mediators and pathways [6]. CEA CD3 TCB (RG7802, RO6958688) is an IgG1 BiTE that simultaneously binds carcinoembryonic antigen (CEA) on tumor cells and CD3 on T cells to increase tumor-infiltrating lymphocytes (TIL) activation, infiltration, and expression of PD-1/PD-L1 [11]. Two ongoing phase I clinical trials using this new drug are currently recruiting patients (NCT02324257, NCT02650713). Preliminary results reveal that the most common adverse events were mild and $16 \%$ of patients develop grade 3 or more adverse events. Five percent and $20 \%$ of patients in each study, respectively, showed partial response (PR), and more importantly, activity appeared to be enhanced if it was combined with the anti-PD-L1 antibody, atezolizumab [11].

Blinatumomab is another BiTE that binds CD3 on T cells as well as CD19 on malignant B cells. The antibody is FDA-approved for the use of Philadelphia chromosomenegative B cell acute lymphoblastic leukemia (B-ALL) [12]. A phase II clinical trial in relapsed/refractory diffuse large B cell lymphoma demonstrated a complete response (CR) rate of $19 \%$ and progression-free survival (PFS) of up to 20 months [13].

BAY2010112 (AMG212, MT112) and MOR209/ES414 are prostate-specific membrane antigen (PSMA)/CD3 BiTEs that are being investigated in phase I clinical trials in patients with castration-resistant prostate cancer (NCT01723475, NCT02262910).

DARTs differ structurally to BiTEs as stated above [5]. MGD009 is a humanized DART protein that binds both T cells and tumor-associated B7-H3 and is being studied in a phase I clinical study in patients with B7-H3 expressing tumors including melanoma, non-small cell lung carcinoma (NSCLC), mesothelioma, and urothelial cancers [14]. The trial is ongoing and recruiting patients (NCT02628535). Flotetuzumab, another DART that binds CD3 and CD123, is currently being studied in a phase I clinical trial in patients with relapsed or refractory acute myeloid leukemia (AML) and intermediate/high-risk myelodysplastic syndrome (MDS) (NCT02152956).

AFM13 is a tetravalent bispecific antibody that is directed against CD30 and CD16A, this latter found over natural killer (NK) cells. Pharmacokinetics, therapeutic index, and efficacy make this agent a NK cell activator [15]. A phase I clinical trial used AFM13 on patients with relapsed/refractory CD30+ Hodgkin's disease (HD) and concluded that three out of 26 patients achieved PR and 13 obtained a SD with an overall disease control rate (DCR) of 61.5\% [16]. Adverse events were mostly mild to moderate and ranged from fever to infusion reactions and pneumonia. A phase II clinical trial using this agent is also being done on patients with HD; however, results have not yet been published (NCT02321592).

\section{Antibody drug conjugates}

Antibody drug conjugates (ADCs), an emerging therapeutic approach in oncology, combine a monoclonal antibody with a high selectivity for specific targets with a cytotoxic agent. Microtubule inhibitors or DNAdamaging chemotherapeutic agents are the two main cytotoxic agents used in ADCs. One of the most important aspects in ADC therapy consists on the appropriate selection of the target antigen. An ideal antigen is one that is overexpressed by malignant cells with very limited or no expression by normal tissue [17]. For example, nectin-4 is often overexpressed in bladder, breast, lung, and pancreatic cancer, and thus, ACDs against this peptide are indicated in these malignancies. Similarly, folate receptor alpha is more often expressed by ovarian and endometrial carcinomas, and CEA cell adhesion molecule (CEACAM) 5 is commonly found on CRC [17]. Another important factor is the conjugate linker, which largely influences the pharmacokinetics and therapeutic efficacy of ADCs [17]. As with other drugs, resistance 
can emerge through different mechanisms such as limitation of intracellular concentration of ADC, downregulation of target antigens, reduction of internalization of $\mathrm{ADC}$, increased ADC recycling to mask the antigen epitopes, or activation of alternative signal pathways. Class side effects are often associated with the linked cytotoxic agents. The major challenges of ADCs include target antigen specificity and drug delivery efficiency.

ABBV-399 is an ADC composed of an anti-c-Met antibody (ABT-700) conjugated to a microtubule inhibitor (monomethyl auristatin E). As the c-Met receptor is commonly overexpressed in patients with NSCLC, a phase I clinical trial is using this agent as monotherapy or in combination with erlotinib in this patient population (NCT02099058). Preliminary results demonstrated adverse events in $>10 \%$ of patients across all grades, $19 \%$ of patients ( 3 out of 16 ) had a PR, and, at week 12, $37.5 \%$ (6 out of 16 ) had disease control including stable disease and partial response [18].

Glembatumumab vedotin (GV, CDX-011) is an ADC that contains an antibody that targets glycoprotein nonmetastatic b (gpNMB), a transmembrane glycoprotein usually overexpressed in melanoma and other tumors, conjugated to monomethyl auristatin E. A phase II clinical trial using this agent as monotherapy in patients with advanced melanoma is recruiting patients (NCT02302339). Preliminary results show 1 CR, 6 PR, and 33 SD out of 62 patients enrolled [19].

Losatuxizumab vedotin (ABBV-221), an ADC that targets EGFR, is being investigated in a phase I clinical trial as monotherapy on patients with EGFR-dependent tumors (NCT02365662). Preliminary results reveal that the most common adverse events were infusion reactions and fatigue and 16 out of 42 patients (38\%) showed SD and 1 patient had an unconfirmed PR [20].

Mirvetuximab soravtansine (IMGN853) is an ADC containing the tubulin inhibitor (maytansinoid) DM4, targeting the folate receptor alpha (FR $\alpha)$. It is being studied in two phase I clinical trials as monotherapy (FORWARD I-NCT01609556) and in combination with bevacizumab, carboplatin, liposomal doxorubicin, or pembrolizumab (FORWARD II-NCT02606305) in patients with ovarian cancer. Preliminary results of FORWARD I reveal an objective response rate (ORR) of $46 \%$ with one patient having a CR and 16 out of 37 patients having PR [21]. FORWARD II preliminary results do not mention efficacy but do show a good safety profile with most adverse events being grade 2 or less [21].

Enfortumab vedotin (ASG-22CE; ASG-22ME), an ADC that targets nectin-4, is in a phase I clinical trial that is investigating its potential role as monotherapy in patients with metastatic urothelial tumors (NCT02091999). Preliminary results show that adverse events occurred in $85 \%$ of patients; however, most were grade 2 or less. ORR was
$40 \%$, CR was seen in 3 out of 68 patients with a median duration of response of 18 weeks, and the median PFS was 17 weeks [22].

Sacituzumab govitecan (IMMU-132) is an ADC against Trop- 2 antigen expressed in many solid tumors and carrying the topoisomerase inhibitor, SN-38. A phase I/II clinical trial as monotherapy in patients with epithelial cell tumors is undergoing (NCT01631552). Preliminary results in triple negative breast cancer show the agent is well tolerated demonstrating an ORR of $30 \%$ with two cases of CR and 19 out of 69 patients demonstrating a PR. Median PFS was 6 months and median OS was 16.6 months [23]. In NSCLC, an ORR of $19 \%$ was observed among the 47 patients, with a median response duration of 6 months, a median PFS of 5.2 months, and a median OS of 9.5 months [24].

Inotuzumab ozogamicin ( $\mathrm{InO} / \mathrm{CMC}-544)$ is a humanized ADC directed against CD22, coupled to a DNA breaking calicheamicin. The compound was recently approved by the FDA for use in relapsed or refractory BALL [25]. This agent was recently evaluated in a phase I clinical trial in conjunction with rituximab, gemcitabine, dexamethasone, and cisplatin in patients with refractory CD22+ non-Hodgkin's lymphoma (NHL) [26]. Results demonstrated an $85 \%$ incidence of thrombocytopenia and a $69 \%$ of neutropenia with an ORR of $53 \%$.

Labetuzumab govitecan (IMMU-130) is an ADC that targets CEACAM 5 which is expressed by $>80 \%$ of CRC [27]. This ADC is being evaluated in a phase II clinical trial in patients with metastatic CRC (NCT01605318). Results reveal that one out of 86 patients enrolled had a PR that extended beyond 2 years, 42 patients had SD, OS was 6.9 months, and PFS was 3.6 months [27].

Lorvotuzumab mertansine (IMGN901), an ADC against CD56 conjugated to the tubulin inhibitor DM1, that was recently studied in a phase I/II clinical trial in combination with carboplatin and etoposide in small cell lung cancer (SCLC) patients with extensive disease [28]. The two-arm cohort of 94 patients (combination ADC with chemotherapy) and 47 patients (no ADC) achieved a PFS of 6.2 and 6.7 months, respectively, with a median OS of 10 months in both cohorts. The ORR was $67 \%$ for the combination cohort compared to $59 \%$ in the noncombination with no statistical significance. Thus, the authors concluded that the combination of lorvotuzumab mertansine did not improve efficacy over standard therapy [28].

Rovalpituzumab tesirine (Rova-T) is an ADC that targets delta-like protein 3 (DLL3) which has been found to be elevated in patients with SCLC. The antibody is conjugated to a DNA cross-linker tesirine, and results from the first-in-human trial in patients with recurrent SCLC showed that 28 out of 74 patients (38\%) developed serious adverse events consisting of pleural and pericardial 
effusions, 18\% demonstrated OR, and 54\% had SD [29]. Other phase I/II clinical trials evaluating this agent are currently ongoing (NCT02674568, NCT03026166).

ADCT-301 is the first ADC against CD25, a receptor for IL-2 often found on hematological tumors that has a role in prognosis and oncogenesis in these malignancies [30]. This molecule is being studied in a phase I clinical trial in patients with relapsed or refractory $\mathrm{HD}$ and NHL (NCT02432235). Preliminary results on 18 patients show 1 $\mathrm{CR}, 1 \mathrm{PR}$, and $6 \mathrm{SD}$, one of which has remained progression-free for over 30 weeks and four developing adverse events consisting of rash, mucositis, enteritis, and elevated creatine phosphokinase [31]. Another phase I clinical trial is being conducted in patients with refractory or relapsing CD25-positive AML and ALL (NCT02588092).

TAK-264 (MLN0264), a novel ADC that targets guanylyl cyclase C (GCC), has been recently studied in a phase II clinical trial in patients with advanced or metastatic pancreatic adenocarcinoma expressing GCC [32]. The cohort of 43 patients achieved an ORR of only $3 \%$, and all patients experience at least one adverse event; thus, the authors concluded these results did not support further clinical investigation of this molecule.

HER2, a well-studied member of the epidermal growth factor tyrosine kinase receptor family, plays an important role in breast cancer and has been a target for ADCs. TDM1 (Kadcyla), an ADC consisting of trastuzumab (T) and a microtubule inhibitor (DM1), was the first ADC approved by the FDA to use in solid tumors [33]. Other anti-HER2 ADCs are being studied in multiple phase I/II clinical trials including MEDI-4276 (NCT02576548), XMT-1522 (NCT02952729), ARX-788 (NCT02512237), DS-8201a (NCT02564900), SDY985 (NCT02277717), and ADCT-502 (NCT03125200).

Glycoprotein non-metastatic b (GPNMB), a highly expressed protein in melanoma and breast cancer, plays an important role with modulation, and is an important target in ADC. Glembatumumab vedotin (CDX-011, CR-11-vc-MMAE) targets GPNMB, and a detailed review on this agent has been recently published [34]. A phase II clinical trial on patients with melanoma (NCT02302339) demonstrated that, while using this ADC, 1 out of 62 patients achieved CR, 6 achieved PR, and 33 had SD, and the median OS was 9.8 months. Toxicities were manageable and included alopecia, neutropenia, and rash [19]. Another phase II clinical trial using glembatumumab vedotin on patients with advanced breast cancer demonstrated an ORR of $6 \%$ with mild toxicities including rash and pruritus [35].

CA6 is a tumor-associated antigen that can be overexpressed in many solid tumors with a low expression on normal tissues [33]. SAR566658 targets CA6 and delivers maytansinoid DM4. On a first-in-human clinical trial on patients with solid tumors expressing CA6 using this agent, 114 patients were enrolled. Results revealed 1 CR, 8 PR, and 39\% SD with overall mild toxicities including fatigue, neuropathy, neutropenia, and gastrointestinal symptoms [36].

LIV-1 is a transmembrane protein that is highly expressed in breast cancer cells. SGN-LIV1A is an antiLIV-1 ADC that is being tested on a phase I clinical trial on patients with metastatic LIV-1-postive breast cancer (NCT01969643). Preliminary results demonstrate no dose-limiting toxicities, an ORR of $11 \%$ and an SD or better achieved in $63 \%$ of patients [37].

PF-06647020 is an ADC directed against protein tyrosine kinase 7 (PTK7) which is overexpressed in a variety of tumors including lung, CRC, breast, and ovarian cancers [33]. This molecule is being studied in a phase I clinical trial on patients with advanced solid tumors (NCT02222922). Preliminary results revealed that 1 out of 76 patients had a CR, 5 had PR, 12 had SD, and 4 had PD. Most toxicities were grades 1-2 and included nausea, alopecia, neutropenia, and gastrointestinal complaints [38].

Ephrin-A4 has been found to be elevated in multiple malignancies including lung, pancreas, and breast [33]. PF-06647263 is an anti-Ephrin-A4 ADC that is coupled to calicheamicin and that was studied in a phase I clinical trial on patients with advanced solid tumors [39]. Results revealed that 5 out of 48 patients achieved PR and that dose-limiting toxicities were observed in 6 patients [39].

LAMP-1 is a protein highly expressed by lysosomes that translocates to the surface of tumor cells, and its expression may influence invasiveness and metastatic behavior including CRC, melanoma, and laryngeal cancers [33]. SAR428926 is an anti-LAMP-1 ADC coupled with DM4 that is currently being studied on a phase I clinical trial in patients with advanced solid tumors (NCT02575781). Preliminary results have not been published.

PCA062 is another ADC that is targeted against Pcadherin 3, which is often overexpressed in epithelial tumors [33]. This agent is being studied in a phase I clinical trial on patients with $\mathrm{P}$-cadherin positive tumors (NCT02375958). No preliminary results have been published.

HER-3 overexpression in breast cancer is associated with poor prognosis, and an anti-HER3 ADC therapy, U3-1402, is being studied in a phase I clinical trial on patients with HER-3-positive metastatic breast cancer (NCT02980341) [40]. Preliminary results have not been published. HuMaxAxl is an ADC against an Axl-specific immunoglobulin (IgG1к) which is present in malignancies like pancreatic, thyroid, and lung cancer and melanoma [41]. A phase I/II clinical trial on patients with solid tumors is studying the use of this agent (NCT02988817); however, preliminary results are not yet available. 
PSMA is highly expressed in prostate cancer, and MEDI3726, an anti-PSMA ADC, is being studied on a phase I/Ib clinical trial in patients with metastatic castration-resistant prostate cancer (NCT02991911). Preliminary results are yet to be published.

\section{Chimeric antigen receptor (CAR) T cells}

CARs are typically genetically engineered $T$ cell receptors with an antibody-based extracellular domain that specifically recognizes a tumor antigen, a transmembrane portion, and an intracellular domain that activates the $\mathrm{T}$ cell. By antigen-specific recognition in a MHCindependent manner, CAR $\mathrm{T}$ cells are activated in vivo through phosphorylation of immune receptor tyrosinebased activation motifs (ITAMs) leading to cytokine secretion, $\mathrm{T}$ cell proliferation, and antigen-specific cytotoxicity. CAR $\mathrm{T}$ cells are produced by inserting specific CAR genes via viral vectors into autologous or allogeneic $\mathrm{T}$ cells [42]. New-generation CARs have two or more co-stimulatory domains (e.g., 4-1BB, OX 40) that boost the stimulatory signal $[43,44]$. Anti-CD19 CAR T cells were recently FDA-approved for B-ALL in pediatric and young adult population [45]. Other CARs rely on the ligand of the receptor of interest rather than on an antibody [46]. Although impressive clinical activities of CAR $\mathrm{T}$ cells in hematological malignancies are reported, several obstacles need to be overcome for a successful application of CAR T cells in solid tumor [47]. Some of these obstacles include the lack of ideal tumor-specific antigens, the inefficient trafficking of CAR $\mathrm{T}$ cells to tumor sites, the immune-suppressive tumor microenvironment, and the risk of developing on-target/off-tumor toxicities which results from an attack to host cells that express the targeted tumor antigen. One of the major challenges with this therapy is the cytokine release syndrome (CRS). This potentially fatal toxicity occurs after a massive release of cytokines and causes complications ranging from mild fever and fatigue to severe respiratory distress, cardiac dysfunction, or even disseminated intravascular coagulation [48]. Future investigation to improve the safety, specificity, and efficiency will likely take CAR $T$ cell therapy into the central stage in cancer immunotherapy [49].

\section{T4 immunotherapy}

T4 immunotherapy uses genetically engineered $\mathrm{T}$ cells that co-express two CARs, T1E28z that targets ErbB dimers, and $4 \alpha \beta$ that binds IL- 4 and promotes T cell expansion [50]. These CAR T cells are currently undergoing a phase I clinical trial on patients with head and neck squamous cell carcinoma (HNSCC) (NCT01818323). Preliminary results show limited adverse events and a disease control rate (DCR) of $44 \%$ [51].

\section{Anti-CD19 CAR T cells (CART-19)}

19-28z CAR (JCAR015) consists of a single-chain murine antibody against human CD19 (expressed by B cell malignancies) fused with the transmembrane and cytoplasmic domains of the human CD28 co-stimulatory molecule [52]. Currently, these CAR T cells have been studied in a phase I clinical trial in patients with relapsed B-ALL (ROCKET Trial-NCT01044069). In this trial, patients were divided into two cohorts, those with $<5 \%$ of blasts in the bone marrow and those with $>5 \%$. CR rates were 95 and $77 \%$, respectively. The duration of this response, however, was directly proportional to the disease burden, and therefore, authors suggest an early use of this therapy before $>5 \%$ of blasts occupy the bone marrow [53].

The ZUMA-1 trial is studying anti-CD-19 CAR T cells (KTE-C19) in patients with refractory aggressive NHL (NCT02348216). Preliminary results reveal an ORR of $82 \%$, and with a median follow-up of 8 months, 39\% remained in CR. Importantly, 43 patients received tocilizumab and 27 out of 111 received steroids; however, ORR did not change significantly with the addition of this therapy [54].

CART-19 are also being studied in other malignancies including Richter syndrome [55] and relapsed chronic lymphocytic leukemia, myeloma, or NHL (NCT01865617). Although in one study $4 \%$ died due to CRS [56], efficacy results seem promising with some patients achieving CR or remission at the time of result publication $[56,57]$.

\section{Anti-GPC3 CAR T cells}

Glypican-3 (GPC3) is a membrane proteoglycan selectively expressed by hepatocellular carcinoma (HCC) cells that is being used as a target CAR therapy [58]. GPC3 CAR T cells were studied in a phase I clinical trial of patients with GPC3+ HCC. Preliminary results showed no dose-limiting toxicities; 1 out of 13 patients had a PR, and 3 showed SD. These responses were seen in patients who received lymphodepletive conditioning [59].

\section{Anti-CD133 CAR T cells (CART-133)}

CD133 is expressed by many tumors of epithelial origin, and therefore, the use of CAR T cells against this molecule (CART-133) is undergoing investigation being in a phase I clinical trial in patients with advanced metastatic malignancies including HCC, pancreatic carcinoma, CRC, and cholangiocarcinoma (NCT02541370). Preliminary results show some grade 3 adverse events consisting of hyperbilirubinemia and CRS. However, 21 out of 23 patients had PFS periods ranging from 8 to 22 weeks. Furthermore, 2 patients maintained greater than 8-month $\mathrm{SD}$ at the time of publication [60]. 


\section{Anti-BCMA CAR T cells (bb2121)}

$B$ cell maturation antigen (BCMA) is expressed by multiple myeloma (MM) cells, and anti-BCMA CARs have been genetically incorporated to $\mathrm{T}$ cells [61]. One of these CAR T cells, bb2121, is currently under investigation in a phase I clinical trial on patients with refractory MM who have $\geq 50 \%$ BCMA expression on their plasma cells (NCT02658929). Preliminary results show only minor adverse events with grade 1 or 2 CRS, and an ORR was seen in all patients (6 out of 11 ) who received the higher doses of $\mathrm{T}$ cells [62].

LCAR-B38 is another CAR T cell that targets BCMA that was recently studied in a phase I clinical trial of patients with refractory or relapsed MM. Preliminary results were encouraging. Seventy-four percent of patients (14 out 19) developed CRS, but most of these were mild in severity. Importantly, ORR and CR/near CR rates were 100 and 95\%, respectively, at a median follow-up of 6 months [57].

\section{Anti-CD138 CAR T cells}

CD138 is a highly expressed molecule on MM cells and has a role in their development and proliferation [63]. A phase I clinical trial using anti-CD138 CAR T cells was conducted in patients with chemotherapy-refractory MM [63]. Five patients were enrolled, of which 4 achieved SD for more than 3 months and 1 with PD. The main toxicity observed was CTS.

\section{Anti-immunoglobulin kappa light chain CAR T cells}

As a way to spare normal cells from being targeted by this therapy, CAR T cells directed against more tumorspecific proteins like kappa light chains are also being developed [64]. A phase I clinical trial testing this therapy on patients with kappa-positive chronic lymphocytic leukemia (CLL), NHL, or MM is currently undergoing (NCT00881920). Results of this trial showed that 2 out of 9 patients with NHL or CLL had CR and 1 had PR. Four of the 7 patients with MM showed SD that lasted 2-17 months. No toxicities were seen with this therapy [64].

\section{Anti-CD30 CAR T cells}

CD30 is expressed in a limited amount on normal tissues whereas is often overexpressed in patients with HD and NHL [65]. The use of anti-CD30 CAR T cells was recently studied in a phase I clinical trial on 9 patients with refractory or relapsed HD and anaplastic large cell lymphoma (ALCL) [66]. No toxicities were observed with this therapy, 2 patients with HD and 1 with ALCL achieved CR, and 3 patients with $\mathrm{HD}$ achieved SD. The use of anti-CD30 CAR T cells in combination with bendamustine is being studied on a phase Ib/II clinical trial on patients with CD30+ HD and NHL (NCT02690545).

\section{Anti-IL13 CAR T cells}

Recently, the use of CAR T cells targeting IL13R $\alpha 2$ in a patient with recurrent multifocal glioblastoma demonstrated CR sustained for 7.5 months with no associated systemic side effects [67].

\section{CARs on the horizon: NKG2D, NKR-2, and mesothelin CAR T cells}

Natural killer group 2D (NKG2D) receptor recognizes specific ligands over tumor cells and promotes $\mathrm{T}$ cell activation to eliminate NKG2D ligand-expressing cells [68]. A first-in-human phase I clinical trial is using genetically modified $\mathrm{T}$ cells expressing NKG2D in patients with AML, MDS, and MM (NCT02203825).

NKR-2 CAR T cells consist of a fusion of NKG2D receptor with CD3 signaling domain, which are under investigation on a phase I clinical trial in patients with both solid and hematologic malignancies (THINK-Therapeutic immunotherapy with NKR-2-trial) (NCT03018405).

Finally, mesothelin as a target for CAR T therapy in patients with mesothelioma has been recently reviewed [69]. Various phase I clinical trials are exploring treatment approach (NCT02414269, NCT01583686, NCT02580747). Results for these future studies have yet to be reported.

\section{T cell receptor (TCR) gene-modified T cell therapy}

In contrast to CAR T cell therapy, TCR gene-modified T cell therapy functions by targeting the surface antigens of tumor cells to specifically recognize intracellular tumor antigens presented by HLA molecules. By genetic transfer of TCR directed against specific tumor antigens into normal $\mathrm{T}$ cells, $\mathrm{T}$ cells are able to perform antigenspecific tumor killing. In addition, genetic engineering of $\mathrm{T}$ cell also offers the advantage of introducing molecules that can enhance $\mathrm{T}$ cell function or overcome tumor escape mechanisms, such as adding genes encoding cytokines, chemokine receptors and costimulatory factors, as well as elements to silence inhibitory molecules. Currently, genes encoding TCRs that are specific for a variety of tumor antigens such as MART-1, gp100, p53, NY-ESO-1, MAGE-A3, and MAGE-A4 have been studied as therapeutic targets for TCR gene-modified $\mathrm{T}$ cell therapy in clinical trials for melanoma, lung cancer, and breast cancer patients [70]. Choosing highly specific tumor antigens, maintaining TCR expression over time, and limiting off tumor/on target toxicity are remaining challenges [70].

\section{Anti-NY-ESO-1 TCR T cells (NY-ESO-1c259t)}

NY-ESO-1 is expressed in up to $70 \%$ of synovial sarcomas and other mesenchymal tumors and the use of TCR 
gene-modified cells, specifically, NY-ESO-1c259T cells that recognize an HLA-A2 peptide are being studied. An ongoing phase I/II clinical trial is recruiting patients with unresectable or metastatic synovial sarcoma who express NY-ESO-1 and HLA-A2 (NCT01343043). Preliminary results demonstrated the development of leukopenia (96\%), anemia (79\%), thrombocytopenia (79\%), one fatal bone marrow failure, and 11 out of 34 cases with CRS. However, ORR was 50\% and 1 patient had a PR [71].

\section{Anti-E6 TCR T cells}

The E6 oncoprotein is an essential component of HPVrelated tumorigenesis, and its expression is maintained in advanced lesion and represents an ideal tumorspecific antigen. TCR gene-modified $\mathrm{T}$ cells that express a TCR that recognize an HLA-A*02:01-restricted epitope of E6 has been developed [72]. A phase I/II clinical trial using these T cells in patients with metastatic HPV 16+ carcinomas, including cervical, anal, and pharyngeal, was recently completed. No dose-limiting toxicity was seen, and 2 out of 12 patients demonstrated PRs [73].

\section{Anti-MAGE A10 TCR T cells}

MAGE-A10 peptide is expressed by different malignancies including urothelial, HNSCC, and melanoma [74]. The use of T cells containing the MAGE-A10c796 CAR is being studied in a first-in-human phase I clinical trial on patients with advanced or inoperable urothelial cancer, HNSCC, or melanoma (NCT02989064). Preliminary results are not available yet.

\section{Tumor-infiltrating $\mathbf{T}$ cell therapy}

Adoptive cell therapy that utilizes endogenous tumorinfiltrating lymphocytes (TIL), which are expanded in vitro from a surgically resected tumor and then re-infused back into the patient, has demonstrated a $20 \%$ complete response lasting beyond 3 years in patients with stage IV melanoma [75]. TILs are naturally occurring T cells in the host able to recognize tumor antigens. This likely explains the highly specific anti-tumor responses and the relatively low toxicity of TILs in comparison with TCR gene-modified T cell therapy and CAR T cell therapy. In addition, TILs are heterogeneous in their specificity which represents an important advantage for impeding immunologic escape. Furthermore, TIL therapy bypasses the limitation identifying specific tumor antigens or the patient's HLA type. Nonetheless, all its clinical advantages are somewhat blunted by the complex process required to generate patient-specific TILs for clinical use. Strategies to improve and simplify the TIL production are being studied.

Infusion of ex vivo expanded TIL is currently being studied in conjunction with total body irradiation (TBI) in a phase II clinical trial in patients with melanoma
(NCT01319565). Preliminary results reveal that 13 out of the 48 patients who received TBI developed thrombotic microangiopathy, but this was not seen in the group without TBI. Regardless, a CR rate was seen in $24 \%$ of patients in both groups, and only one of these patients had recurrence of the disease [76].

Marrow-infiltrating lymphocytes (MILs) have been used in patients with newly diagnosed or relapsed MM. In a phase I clinical trial, the overall clinical response was of $54 \%$, with $27 \% \mathrm{CR}$ and $27 \%$ PR, $23 \%$ of patients had a SD, and only $14 \%$ showed a PD. Patients who achieved at least $90 \%$ reduction of disease burden had a PFS almost 13 months longer; however, no difference in OS was seen [77].

\section{Oncolytic viruses}

Native or genetically modified viruses are a new therapeutic approach within the immunotherapy spectrum. The mechanisms of action of oncolytic viruses are not fully elucidated but likely depend on viral replication within tumor cells, induction of primary cell death, interaction with tumor cell antiviral elements, and initiation of innate and adaptive anti-tumor immunity. A variety of native and genetically modified viruses have been developed as oncolytic agents [78]. Of note, these viruses selectively infect malignant cells due to the lack of adequate function of anti-viral mechanisms. Though many viruses have been considered, the most widely studied to date include herpes simplex virus type 1 (HSV-1), coxsackievirus, reovirus, and adenovirus.

Talimogene laherparepvec (T-VEC; Imlygic) is the first oncolytic virus approved by the FDA for its use in melanoma. It is an attenuated HSV-1 engineered to replicate within tumor cells and enhance immune responses [79]. Treatment has been relatively well tolerated, with the major side effects including fever, chills, nausea, fatigue, and reaction of local injection site.

Coxsackievirus A21 (CVA21-CAVATAK) preferentially infects tumors that express ICAM-1. This virus increases TIL and PD-L1 expression [80]. Therefore, the use of CVA21 is currently being studied in conjunction with pembrolizumab in two phase Ib clinical trials in patients with solid tumors including melanoma, NSCLC, bladder, and prostate cancer (NCT02043665, NCT02565992). Both STORM [81] and CAPRA [82] trials demonstrated that the combination is generally well tolerated and the latter also showed an ORR of $73 \%$ and a DCR of $91 \%$ of patients with advanced melanoma. CVA21 is also being studied in conjunction with ipilimumab in patients with unresectable melanoma (NCT02307149). Preliminary results demonstrate minimal additional toxicity with an ORR of $38 \%$ (3 out of 8 patients) and a DCR of $88 \%$ (7 out of 8 patients) [83]. 
Pelareorep (Reolysin) is a strain of reovirus serotype-3 which has shown in vitro and in vivo activity against many cancers and synergistic activity with concomitant use of microtubule-targeting drugs [84]. This agent has been studied in a phase II clinical trial in combination with carboplatin and paclitaxel for patients with advanced malignant melanoma. Results revealed and ORR of $21 \%$, no CR, and a 1year survival of $43 \%$ with a DCR of $85 \%$ [85]. Another phase II clinical trial using pelareorep with paclitaxel versus paclitaxel alone in patients with metastatic breast cancer was also recently published [84]. Median OS was 17.4 months for patients with both agents and 10.4 months for patients with paclitaxel alone; however, PFS was not different between the groups.

The oncolytic adenovirus DNX-2401 was also studied in a phase I clinical trial using temozolamide in patients with first recurrence of glioblastoma [86]. One patient out of 31 was still alive 30 months after the treatment was started, and 2 other patients were still alive 23 months after the agent was given.

Enadenotucirev (EnAd) is an A11/Ad3 chimeric group $\mathrm{B}$ oncolytic adenovirus that is currently under investigation in combination with nivolumab for patients with tumors of epithelial origin such as salivary gland, urothelial, HNSCC, and CRC (NCT02636036). No preliminary results have yet been published.

\section{Vaccines}

Therapeutic vaccines are designed to increase immune response against malignant cells by enlarging antigen-specific $T$ cell from endogenous $T$ cell repertoire. Its use has the advantage of producing a specific immune response that potentially spares normal cells [87]. Successful cancer vaccines require the selection of appropriate antigens, a platform able to induce robust effector and memory $\mathrm{T}$ cell responses, and strategies to overcome immune evasion and suppression. Although cancer vaccines can be effective in settings of early cancer or minimal residual disease, therapeutic cancer vaccines will most likely require cotreatment, such as immune checkpoint inhibitors, to overcome immune suppression and be clinically effective in established cancers.

Depending on its composition, vaccines can be classified into tumor cell vaccines (autologous/allogenic), genetic vaccines (DNA/RNA/viral/bacterial), dendritic cell (DC) vaccines, and protein/peptide vaccines.

\section{Tumor cell vaccines}

Tumor cell vaccines are classified as autologous when patient-derived tumor cells are used, or allogenic if established human tumor cell lines are the utilized. Among the allogenic vaccines, HS-110 (viagenpumatucel-L) is derived from lung adenocarcinoma cells. This vaccine is currently being studied in combination with nivolumab on a phase I/IIb clinical trial (DURGA trial) which is recruiting patients with NSCLC (NCT02439450). Preliminary results showed ORR of $50 \%$ in patients with IR (immune response, defined by doubling of IFN $\gamma$-secreting cells after re-stimulation with total vaccine antigen and individual cancer antigens) in comparison to $0 \%$ in patients without immune response among the initial treated 8 patients. Interestingly, patients with objective responses were also found decrease in MDSC and increase in CD8+ T cells in the blood [88].

An autologous tumor-derived gp96 vaccination was studied in a phase II clinical trial in patients with advanced gastric cancer [89]. The trial enrolled 73 patients of which 38 received both vaccination and chemotherapy and the remainder received chemotherapy alone. Overall vaccination was well tolerated with no clinically significant adverse events. The 2 -year OS was $81.9 \%$ in the vaccination group compared to $67.9 \%$ in the chemotherapy-alone arm, though this was not statistically significant [89].

The GM.CD40L is another allogenic vaccine composed of radiated lung adenocarcinoma cells transduced with the GM-CSF and CD40-ligand (CD40L) genes. This vaccine is being studied in patients with lung adenocarcinoma in a phase I/II clinical trial in combination with nivolumab (NCT02466568) and in combination with CCL21, a chemokine that enhances $\mathrm{T}$ cell response (NCT01433172). Preliminary results reveal an acceptable safety profile with no dose-limiting toxicities and a median OS similar to single-agent nivolumab (9.4 months) [90].

\section{Genetic vaccines}

Genetic vaccines use DNA/RNA plasmids, bacteria, or viruses to deliver antigens by transfection of cells that subsequently process them and present them to immune cells [91].

The use of messenger RNA (mRNA) that encodes tumor antigens is currently being studied in clinical trials. RNA-Lipoplex $\left(\mathrm{RNA}_{(\mathrm{LIP})}\right)$ is a mRNA vaccine that encodes melanoma antigens (e.g., NY-ESO-1). It is being studied in a first-in-human phase I/II (Lipo-MERIT) clinical trial (NCT02410733). Preliminary results in 15 patients show no dose-limiting toxicities, but no efficacy results have been published yet [92].

VXM01 is an oral vaccine derived from live, attenuated Salmonella carrying a DNA plasmid that encodes vascular endothelial growth factor receptor-2 (VEGFR2 ). A phase I clinical trial using this vaccine in patients with advanced pancreatic carcinoma showed that 12 out of the 18 patients studied had a considerable increase of specific anti-VEGFR2 T cells and the OS was 9.3 months 
compared to 8.4 months in those who received placebo. Furthermore, patients who showed $\mathrm{T}$ cell response had a longer median OS (10.3 months) compared to those without it (5.4 months) [93].

INO-5150 is a plasmid-based DNA vaccine that encodes for highly expressed prostate cancer antigens with amino acid sequence changes to break immune tolerance. This vaccine is being investigated with and without co-administration of IL-12 (INO-9012) in a phase I clinical trial (NCT02514213). Preliminary results revealed no dose-limiting toxicities and PSA was stable in some patients, whereas $10 \%$ reported disease progression [94].

INVAC-1 is another plasmid DNA vaccine that encodes an inactive form of human telomerase, which is expressed in over $85 \%$ of human tumors [95]. This vaccine is currently being studied in a phase I clinical trial with refractory and progressive solid tumors (NCT02301754). Preliminary results reveal no dose-limiting toxicities with only mild adverse events, 12 out of 20 patients achieved $\mathrm{SD}$, and anti-human telomerase activity was found in $55 \%$ of patients [95].

pTVG-HP, also a plasmid DNA vaccine, encodes prostatic acid phosphatase (PAP). It is being studied in a phase II clinical trial in patients with non-castrate, nonmetastatic prostate cancer (NCT01341652).

Listeria monocytogenes can survive in the cytosol of host cells and is therefore considered an ideal vector for tumor antigens [96]. ADXS11-001 is a live, attenuated Listeria monocytogenes, which is bioengineered to secrete a HPV-E7 antigen, a marker of HPV transformed cells. This vaccine is currently being investigated in a phase I clinical trial in patients with persistent, recurrent, or metastatic cervical carcinoma (NCT02164461). Preliminary results revealed that most adverse events were mild (grades 1 and 2), with no grade 4 or 5 reported. Tumor response analysis is ongoing and not yet published [97].

Adenovirus is also used as a vaccine vector. In a phase I clinical trial, the use of the cancer antigen MAGE-A3 primed to an adenovirus (AdMA3) is being investigated in conjunction with an oncolytic virus (MG-1 Maraba virus) that also expresses the MAGA-A3 antigen (MG1MA3), in patient with MAGE-A3 expressing solid tumors (NCT02285816). Preliminary results reveal a potent induction of pro-inflammatory genes with subsequent anti-tumor activity. However, dose-limiting toxicities also occurred in 4 out of 41 patients manifested by hypoxia, dyspnea, vomiting, and headache [98].

\section{Dendritic cell vaccines}

DCs play an important role in bridging innate and adaptive immunity, and as such are considered an important target for immunotherapy. The autologous tumor lysate, particle-loaded, dendritic cell (TLPLDC) vaccine consists of DCs that are exposed to autologous tumor antigens, become particle-loaded, and are infused back to the patient. This vaccine is being investigated in patients with stage III or IV of ovarian cancer and preliminary results reveal minimal toxicity, 1 out of 12 patients demonstrated CR, 1 had a SD, and 4 had a progression of their disease [99]. AdHER2ECTM consists of autologous DCs expressing human HER2 extracellular and transmembrane domains and is currently under investigation in a phase I clinical trial on patients with advanced tumors expressing HER-2 (e.g., colon, breast, ovarian) (NCT01730118). Preliminary results show that adverse events were limited to local injection site reactions, $37 \%$ had evidence of response, namely, 1 out of the 27 evaluated patients demonstrated CR, 1 had PR, and 5 had SD [100].

CMB305 is another DC vaccine that carries the NYESO-1 gene and a boost with G305, a NY-ESO-1 protein vaccine [101]. This vaccine is currently being investigated in a phase I clinical trial in patients with NY-ESO1 -expressing solid tumors. Preliminary results reveal an increase of anti-NY-ESO- $1 \mathrm{~T}$ cells in up to $65 \%$ of patients and anti-NY-ESO-1 antibodies in up to $68 \%$ of patients [101].

A Modified Vaccinia Ankara (MVA) vector vaccine expressing genes for brachyury (a transcription factor important in the epithelial-to-mesenchymal transition and in tumor resistance to treatment) and costimulatory molecules (e.g., ICAM-1) designated TRICOM was developed for transducing DCs [102]. This vaccine was studied in a phase I clinical trial in 38 patients with advanced solid malignancies [102]. No dose-limiting toxicities were observed, and $82 \%$ of patients developed brachyury-specific immune responses.

BPX101 is another DC-derived vaccine which was recently evaluated in a phase I clinical trial in 18 men with progressive metastatic castrate-resistant prostate cancer [103]. Results revealed no dose-limiting toxicities, one case of CR, and two PR.

\section{Protein/peptide-based vaccines}

The use of specific tumor-associated antigens has the advantage of inducing specific immune response against defined antigens, sparing healthy tissue. However, because only defined epitopes are used, a specific but sometimes insufficient response may be generated as tumor cells often exhibit mutations of the epitopes used [104].

Wilms tumor gene-1 (WT-1) is overexpressed in many hematological and solid malignancies where it plays an oncogenic role [105]. The use of WT-1 peptide vaccine with gemcitabine was investigated in a phase II clinical trial on patients with pancreatic adenocarcinoma [106]. Results revealed an increased OS from 21.5 to 34.2 months in patients with gemcitabine alone 
compared to gemcitabine and WT-1 peptide vaccine, respectively. WT4869 is another peptide vaccine derived from WT1 that was recently studied in a phase I/II clinical trial in 26 patients with MDS. Results reveal an ORR of $18.2 \%$ and a median OS of 64.71 weeks, with an antiWT1 lymphocyte induction occurring in 11 patients [107].

Galinpepimut-S is a WT-1-derived peptide vaccine with GM-CSF and Montanide as adjuvants that is being studied with compared to these last two alone, in a phase II clinical trial in 41 patients with pleural mesothelioma [108]. Results revealed a PFS of $45 \%$ in the patients with the vaccine arm compared to $33 \%$ in those without the vaccine. Median OS was 22.8 months in the vaccine group compared to 18.3. Most adverse events were not clinically significant. Unfortunately, the trial did not achieve statistical power.

DPX-Survivac is a peptide-based vaccine containing survivin epitopes to elicit cytotoxic $\mathrm{T}$ cell response against survivin-expressing tumors. A phase Ib clinical trial on patients with ovarian/fallopian/peritoneal cancer studied this vaccine in combination with low-dose of cyclophosphamide [109]. Besides being safe, it demonstrated achieving sustained immune responses. This vaccine is also being investigated in hematologic malignancies in a phase II clinical trial that is currently recruiting patients (NCT02323230).

AE37 is a vaccine that contains HER-2-derived epitopes that stimulate $\mathrm{T}$ cell response. It is currently being studied on a phase II clinical trial on patients in combination with GM-CSF in patients with HER-2-positive breast cancer (NCT00524277). Preliminary results reveal the vaccine is safe and well tolerated, and disease-free survival was improved from $51 \%$ with the use of GMCSF alone compared to $89 \%$ with AE37+ GM-CSF [110].

Heat shock protein 70 (HSP70) and a glypican-3 (GPC-3)-derived peptide is undergoing investigation in a phase I clinical trial in solid tumors that express these antigens (UMIN000020440). Preliminary results revealed no severe adverse events, a decrease tumor marker expression in 6 out of 12 patients, and disease control in 5 patients was observed [111].

URLC10-CDCA1-KOC1 multipeptide vaccine uses three HLA-A-24-restricted epitope peptides derived from cancer cells: upregulated lung cancer 10 (URLC10), cell division cycle-associated 1 (CDCA1), and $\mathrm{KH}$ domain-containing protein overexpressed in cancer 1 (KOC1). A phase II clinical trial studied this vaccine on patients with esophageal squamous cell carcinoma and found that it is capable of inducing highly specific $T$ cells against these antigens [112].

Other peptide-based vaccines that are undergoing clinical trials but without preliminary results include the mutation-derived tumor antigen (MTA)-based peptide vaccine (NCT02721043); a personalized neoantigen vaccine NEO-PV-01 (NCT02897765); a vaccine composed of GM-CSF and CD40L (GM.CD40l) (NCT02466568); the OCV-C01 composed of peptides derived from KIF20A, VEGFR1, and VEGFR2 (UMIN000007991) [113]; and a Toll-like receptor-2 (TLR2) ligand-synthetic long peptide (SLP) vaccine containing HPV-16 E6 protein long peptides (2014-00065812) [114].

\section{In situ vaccines}

Poly-ICLC is a synthetic immune danger signal and is specifically a mimic of viral dsRNA that can ligate TLR3 and trigger cytokine production by DCs with subsequent immune activation and enhancement of the vaccine-induced anti-tumor responses [115]. A phase I/ II clinical trial was recently conducted using this TLR ligand as an in situ vaccine in patients with multiple solid tumors including melanoma, breast, and HNSCC. Most of adverse events were mild and limited to the site of application; only 1 out of 8 patients achieved SD for 41 weeks, the remainder of patients showed PD [116].

BO-112 is a synthetic dsRNA administered intratumorally; it activates pro-apoptotic signals MDA-5 and NOXA and increases IFN response genes leading to the anti-tumor activity [117]. Its use is being studied in a phase I clinical trial in patients with palpable malignant tumors including melanoma and breast cancer (NCT02828098). Preliminary results revealed only one episode of reversible thrombocytopenia, with increase in circulating immune cells [117].

\section{Neoantigen vaccines}

Neoantigens are molecules expressed on tumor cell's surface by DNA mutations that present in tumor cells, but not in normal cells, making it an attractive cancer vaccine target [118]. Although neoantigen cancer vaccines have been long envisioned as ideal, its discovery and evaluation only became feasible recently with the development of highly efficient sequencing. Different from other immunotherapy such as checkpoint inhibitors and CAR $\mathrm{T}$ cells, vaccines targeting neoantigens are designed to be individual-specific. This personal vaccine induces a focused $\mathrm{T}$ cell response to patient's specific tumor neoantigens and avoids toxicities caused by damage to normal cells and tissues [119]. Multiple studies are ongoing to further explore this novel exciting approach.

A first-in-human clinical trial on patients with advanced melanoma identified individual mutations and neoantigens and developed a vaccine unique to each patient (IVAC MUTANOME) (NCT02035956). All 13 patients showed $\mathrm{T}$ cell response against neoantigens. Eight patients remained recurrence-free for the entire follow-up period (12-23 months). Two of 5 patients that 
relapsed and achieved objective clinical response, and one of them achieved a CR in multiple metastatic lesions that had been unresponsive to radiation therapy and CTLA-4 blockade. A third patient also achieved CR to vaccination in combination with $\mathrm{PD}-1$ blockade [119]. No major adverse events were reported.

The use of specific neoantigens in personalized vaccines is being explored in a phase I clinical trial in patients with melanoma (NCT01970358). The vaccine targets up to 20 personal tumor neoantigens and preliminary results reveal that 4 of the 6 vaccinated patients had no recurrence of the disease at 25 months after vaccination. The other two patients that experienced recurrence were subsequently managed with anti-PD-1 and achieved a complete tumor regression. Adverse events were mild and consisted of flu-like symptoms, injection site reactions, rash, and fatigue [120].

\section{Other approaches in immunotherapy Targeting myeloid-derived suppressor cells}

Myeloid-derived suppressor cells (MDSCs) are immature myeloid cells that promote immunosuppression and favor tumor growth [121]. The TNF-related apoptosisinducing ligand receptor (TRAIL-R)-2, also known as death receptor (DR)-5, is found on tumor cells and MDSCs, and its activation promotes apoptosis in these populations [122]. The use of the TRAIL-R2 agonist antibody, DS-8273a, is being studied. Results of one trial in patients with solid tumors revealed only mild to moderate adverse events, no dose-limiting toxicities, and a decrease in blood levels of MDSCs [123].

\section{Cytokine gene therapy}

IL-12 has been considered a good option for immunotherapy given its potent anti-tumor effect [124]. This cytokine promotes the activation of $\mathrm{NK}$ and $\mathrm{T}$ cells and synergizes other cytokines with anti-tumor effects [124].

Ad-RTS-hIL-12 is a replication-incompetent adenovirus engineered to express IL-12. By default, IL-12 expression by this virus is "off," but with the use of veledimex, gene is activated and $\mathrm{LL}-12$ production is started [125]. The use of Ad-RTS-hIL-12 with veledimex is being studied in patients with advanced gliomas in a phase I clinical trial (NCT02026271). Preliminary results revealed that the most frequent adverse events were mild flu-like symptoms, grade 3 CRS, and grade 3 transaminitis; however, all were reversed upon discontinuation of therapy. A median OS of 12.5 months was also observed [126]. This technology is also being studied in patients with locally advanced or metastatic breast cancer (NCT02423902); however, no preliminary results have been revealed yet.

IL-2 enhances the immune system through the IL-2 receptor (IL-2R) [127]. NKTR-214, an engineered cytokine that specifically stimulates IL-2R, is being investigated on phase I/II clinical trials on solid tumors (NCT02983045, NCT02869295). Preliminary results on the former trial show no dose-limiting toxicities. One patient had a $40 \%$ decrease in LDH, and another patient had an unconfirmed CR after only 6 weeks of treatment [128]. The latter trial revealed no dose-limiting toxicities, a tumor size reduction ranging from 10 to $30 \%$ in 6 out of 26 patients (23\%), and an increase of T cells and NK cells within the tumor microenvironment in $100 \%$ of patients [129].

\section{Targeting tumor microenvironment}

Cancer cells require a milieu, known as tumor microenvironment, which allows their growth. This microenvironment consists of immune and nonimmune cells and non-cellular factors that interact among each other and promote a chronic inflammatory, immunosuppressive, and pro-angiogenic ecosystem that favors tumor survival, growth, and dissemination [130]. Some of these factors that have been identified are being investigated as potential therapeutic targets, often in conjunction with other immunotherapy agents.

Indoleamine 2,3-dioxygenase (IDO) is an enzyme that converts tryptophan to kynurenines. These latter promote the formation of Tregs, increase the number of MDSCs, and decrease the activity of CD8 T cells with a resulting inhibitory environment $[130,131]$. BMS986205 is an IDO1 inhibitor that is being studied on a phase I clinical trial in conjunction with a PD-1 inhibitor in patients with advanced solid tumors (NCT02658890). Preliminary results reported mild toxicities except for three cases of grade 3 hepatitis, rash, and hypophosphatemia. No efficacy was described [132]. Indoximod is another IDO inhibitor undergoing phase II clinical trials on melanoma (NCT02073123) and pancreatic (NCT02077881) and castrate-resistant prostate cancer (NCT01560923). Preliminary results reveal an ORR of $52 \%$ in patients with melanoma when used with immune checkpoint inhibitors [133]. Patients with pancreatic cancer had an ORR of $37 \%$ when indoximod was used with both gemcitabine and nabpaclitaxel [134]. Median PFS increased from 4.1 to 10.3 months in castrate-resistant metastatic prostate cancer compared to placebo [135]. Epacadostat also blocks IDO pathway and is being evaluated on phase I/II clinical trials with multiple solid malignancies (NCT02327078, NCT02178722). Preliminary results have demonstrated an ORR ranging from $75 \%$ in melanoma to $4 \%$ in CRC. No dose-limiting toxicities were identified [136, 137].

Toll-like receptors (TLRs) are critical in the identification of pathogens but play a complex role in 
tumorigenesis. TLRs like TLR4 promote cancer progression by promoting inflammation in the microenvironment. TLRs like TLR7/8 and TLR9 promote anti-tumor responses by inducing a "danger signal" and activating the immune system against malignant cells [138]. MEDI9197, a dual agonist of TLR7/8, is under phase I clinical testing in combination with durvalumab and radiation therapy on metastatic or locally advanced solid malignancies (NCT02556463). Preliminary results show that the agent is overall safe with only mild adverse events. No efficacy data has been yet reported [139]. PG545 (pixatimod, pINN) is an agonist of TLR9/IL-12 tested in a phase I clinical trial in patients with advanced solid tumors [140]. Results show that 3 out of 23 patients developed dose-limiting toxicities and the best response achieved was a $24-$ week SD and a DCR of $38 \%$. Polyinosinic-polycytidylic acid polylysine carboxymethylcellulose (poly-ICLC) is a potent TLR3 agonist that was studied in combination with radiation in a phase I clinical trial in patients with hepatocellular carcinoma not eligible for surgery [141]. Intratumoral injection was found to be safe with mostly grade I-II adverse events, a PFS of $66 \%$ at 6 months and $28 \%$ at 24 months and an OS after 1 year was $69 \%$ and $38 \%$ after 2 years [141].

Arginine is an amino acid required for $\mathrm{T}$ cell activation and proliferation. Malignant cells produce high levels of arginase and deplete arginine interfering with immune activation [142]. CB-1158, an arginase inhibitor, is being studied in a phase I clinical trial alone and in combination with a PD-1 inhibitor in patients with advanced solid tumors (NCT02903914). Preliminary results reveal no dose-limiting toxicities, $>90 \%$ of arginase inhibition, and up to a 4-fold increase in plasma arginine levels [143].

\section{Oncolytic peptides}

LTX-315 is a cytotoxic peptide that damages the tumor-mitochondrial membranes and triggers caspaseindependent necrosis leading to a massive release of tumor antigens and to an increase in TIL activity [144]. A phase I clinical trial is investigating this agent as monotherapy or in combination with ICIs in patients with metastatic solid tumors, particularly melanoma and breast cancer (NCT01986426). Preliminary results showed that 2 patients achieved a CR, 5 patients had a decrease of $>50 \%$ of the tumor size, and 8 patients achieved SD [145].

\section{Conclusions}

Cancer immunotherapy has changed the landscape of modern oncology in varied cancer types. Immunotherapy with checkpoint inhibitors has significantly improved the clinical outcomes in some, but not all patients. This is likely due to individual differences in immunogenicity of tumor and immunosuppressive tumor microenvironments. The understanding of emerging novel immunotherapeutic approaches beyond immune checkpoints discussed above will likely open the opportunities to patients with cancers that have failed to respond to an immune checkpoint inhibitor alone. Furthermore, combination therapies targeting different immune mechanisms will likely to better modulate the immune systems to boost an anti-tumor response.

The development of tumor-directed antibodies, antibody-drug conjugates, CAR T cells, oncolytic viruses, vaccines, and even genetic therapy has allowed for a more targeted and tumor-specific therapy rather than a non-specific cytolytic chemotherapy or radiation therapy. Next wave of clinical trials are already evaluating the combinations of immunotherapy agents from different classes. Immune-related side effects, cost of treatment, lack of response biomarkers, and tumor relapse are remaining challenges. Nevertheless, this rapidly advancing field is becoming the most promising treatment component of current oncologic therapy.

\section{Abbreviations}

AARC: American Association of Cancer Research; ACT: Adoptive cell transfer; ADC: Antibody drug conjugate; ALL: Acute lymphoid leukemia; AML: Acute myeloid leukemia; ASCO: American Society of Clinical Oncology; B-ALL: B cell acute lymphoblastic leukemia; BCMA: B cell maturation antigen;

BiTEs: Bispecific T cell engaging antibodies; CAR: Chimeric antigen receptor; CEA: Carcinoembryonic antigen; CEACAM: CEA cell adhesion molecule; CLL: Chronic lymphocytic leukemia; CR: Complete response; CRC: Colorectal; CTLA-4: Cytotoxic T lymphocyte-associated molecule-4; DART: Dual affinity re-targeting; DC: Dendritic cells; DCR: Disease control rate; GCC: Guanylyl cyclase C; GPC-3: Glypican-3; gpNMB: Glycoprotein non-metastatic b; HCC: Hepatocellular carcinoma; HNSCC: Head and neck squamous cell carcinoma; HSP70: Heat shock protein 70; LAG-3: Lymphocyte activation gene 3; MDS: Myelodysplastic syndrome; MDSCs: Myeloid-derived suppressor cells; MILs: Marrow-infiltrating lymphocytes; MM: Multiple myeloma; MTAs: Mutation-derived tumor antigens; MVA: Modified Vaccinia Ankara; NHL: Non-Hodgkin's lymphoma; NK: Natural killer; NKG2D: Natural killer group 2D; NSCLC: Non-small cell lung carcinoma; OR: Objective response; ORR: Objective response rate; OS: Overall survival; PD-1: Programmed cell death receptor-1; PD-L1: Programmed cell death ligand-1; PFS: Progressionfree survival; PR: Partial response; PSMA: Prostate-specific membrane antigen; SCLC: Small cell lung cancer; SD: Stable disease; TBI: Total body irradiation; TCR: T cell receptor; TILs: Tumor-infiltrating lymphocytes; TIM-3: T cell immunoglobulin; TLPLDC: Tumor lysate, particle-loaded, dendritic cell; TLR: Toll-like receptor; URLC10: Upregulated lung cancer 10; VEGFR2: Vascular endothelial growth factor receptor-2; VISTA: V-domain Ig suppressor of T cell activation; WT-1: Wilms tumor gene-1

\section{Acknowledgements}

Not applicable

Funding

Not applicable

Availability of data and materials

All data generated or analyzed during this study are included in this published article (and its supplementary information files).

\section{Authors' contributions}

JMA drafted the manuscript; $Y L$ designed and supervised the study, as well as edited the manuscript; AS, BD, and KK reviewed and edited the text. All authors reviewed and approved the final version of the manuscript. 


\section{Ethics approval and consent to participate}

Not applicable

\section{Consent for publication}

Not applicable

\section{Competing interests}

The authors declare that they have no competing interests.

\section{Publisher's Note}

Springer Nature remains neutral with regard to jurisdictional claims in published maps and institutional affiliations.

\section{Author details}

${ }^{1}$ Department of Internal Medicine, Mayo Clinic, Jacksonville, FL, USA. 2Department of Hematology and Oncology, Mayo Clinic, 4500 San Pablo Road, Jacksonville, FL 32224, USA. ${ }^{3}$ Current address: Department of Blood and Marrow Transplant and Cellular Immunotherapy, Moffitt Cancer Center Tampa, FL, USA. ${ }^{4}$ Department of Immunology, Mayo Clinic, Jacksonville, FL, USA.

Received: 1 November 2017 Accepted: 28 December 2017 Published online: 12 January 2018

\section{References}

1. Khalil DN, Smith EL, Brentjens RJ, Wolchok JD. The future of cancer treatment: immunomodulation, CARs and combination immunotherapy. Nat Rev Clin Oncol. 2016;13:273-90.

2. Dholaria B, Hammond W, Shreders A, Lou Y. Emerging therapeutic agents for lung cancer. J Hematol Oncol. 2016:9:138.

3. Sharma P, Allison JP. The future of immune checkpoint therapy. Science 2015;348:56-61.

4. Scott AM, Allison JP, Wolchok JD. Monoclonal antibodies in cancer therapy. Cancer Immun. 2012;12:14

5. Weidle UH, Kontermann RE, Brinkmann U. Tumor-antigen-binding bispecific antibodies for cancer treatment. Semin Oncol. 2014;41:653-60.

6. Zhang X, Yang Y, Fan D, Xiong D. The development of bispecific antibodies and their applications in tumor immune escape. Exp Hematol Oncol. 2017;6: 12

7. Fan G, Wang Z, Hao M, Li J. Bispecific antibodies and their applications. J Hematol Oncol. 2015:8:130

8. Moore PA, Zhang WJ, Rainey GJ, et al. Application of dual affinity retargeting molecules to achieve optimal redirected T-cell killing of B-cell lymphoma. Blood. 2011:117:4542-51.

9. Rader C. DARTs take aim at BiTEs. Blood. 2011;117:4403-4

10. Kim RD, Arlen PM, Tsang KY, et al. Ensituximab (E) in patients (pts) with refractory metastatic colorectal cancer $(\mathrm{mCRC})$ : results of a phase I/II clinical trial. J Clin Oncol. 2017:35:3081.

11. Tabernero J, Melero I, Ros W, et al. Phase la and lb studies of the novel carcinoembryonic antigen (CEA) T-cell bispecific (CEA CD3 TCB) antibody as a single agent and in combination with atezolizumab: preliminary efficacy and safety in patients with metastatic colorectal cancer (mCRC). J Clin Oncol. 2017:35:3002

12. Wu J, Fu J, Zhang M, Liu D. Blinatumomab: a bispecific T cell engager (BiTE) antibody against CD19/CD3 for refractory acute lymphoid leukemia. J Hematol Oncol. 2015;8:104

13. Viardot A, Goebeler ME, Hess G, et al. Phase 2 study of the bispecific T-cell engager (BiTE) antibody blinatumomab in relapsed/refractory diffuse large B-cell lymphoma. Blood. 2016:127:1410-6.

14. Tolcher AW, Alley EW, Chichili G, et al. Phase 1, first-in-human, open label, dose escalation study of MGD009, a humanized B7-H3 x CD3 dual-affinity re-targeting (DART) protein in patients with B7-H3-expressing neoplasms or B7-H3 expressing tumor vasculature. J Clin Oncol. 2016;34:TPS3105.

15. Wu J, Fu J, Zhang M, Liu D. AFM13: a first-in-class tetravalent bispecific antiCD30/CD16A antibody for NK cell-mediated immunotherapy. J Hematol Oncol. 2015:8:96.

16. Rothe A, Sasse S, Topp MS, et al. A phase 1 study of the bispecific antiCD30/CD16A antibody construct AFM13 in patients with relapsed or refractory Hodgkin lymphoma. Blood. 2015;125:4024-31.

17. Diamantis N, Banerji U. Antibody-drug conjugates-an emerging class of cancer treatment. Br J Cancer. 2016;114:362-7.
18. Angevin E, Strickler JH, Weekes CD, et al. Phase I study of ABBV-399, a c-Met antibody-drug conjugate (ADC), as monotherapy and in combination with erlotinib in patients (pts) with non-small cell lung cancer (NSCLC). J Clin Oncol. 2017:35:2509.

19. Ott PA, Pavlick AC, Johnson DB, et al. A phase II study of glembatumumab vedotin (GV), an antibody-drug conjugate (ADC) targeting gpNMB, in advanced melanoma. J Clin Oncol. 2017;35:109.

20. Calvo E, Cleary JM, Moreno V, et al. Preliminary results from a phase 1 study of the antibody-drug conjugate ABBV-221 in patients with solid tumors likely to express EGFR. J Clin Oncol. 2017;35:2510.

21. O'Malley DM, Moore KN, Vergote I, et al. Safety findings from FORWARD II: a phase $1 \mathrm{~b}$ study evaluating the folate receptor alpha (FRa)-targeting antibody-drug conjugate (ADC) mirvetuximab soravtansine (IMGN853) in combination with bevacizumab, carboplatin, pegylated liposomal doxorubicin (PLD), or pembrolizumab in patients (pts) with ovarian cancer. J Clin Oncol. 2017;35:5553.

22. Petrylak DP, Perez RP, Zhang J, et al. A phase I study of enfortumab vedotin (ASG-22CE; ASG-22ME): updated analysis of patients with metastatic urothelial cancer. J Clin Oncol. 2017:35:106.

23. Bardia A, Mayer IA, Diamond JR, et al. Efficacy and safety of anti-Trop-2 antibody drug conjugate sacituzumab govitecan (IMMU-132) in heavily pretreated patients with metastatic triple-negative breast cancer. J Clin Oncol. 2017:35:2141-8.

24. Heist RS, Guarino MJ, Masters G, et al. Therapy of advanced non-small-cell lung cancer with an SN-38-anti-Trop-2 drug conjugate, Sacituzumab Govitecan. J Clin Oncol. 2017;35:2790-7.

25. Pazdur R. Inotuzumab ozogamicin (Besponsa) approval letter. In: Drug approvals and Databases. U.S. Food and Drug Administration, Center for Drug Evaluation and Research. 2017. https://www.accessdata.fda.gov/ drugsatfda_docs/appletter/2017/7610400rig1 s000ltr.pdf. Accessed 3 October 2017

26. Sangha $\mathrm{R}$, Davies A, Dang NH, et al. Phase 1 study of inotuzumab ozogamicin combined with R-GDP for the treatment of patients with relapsed/refractory CD22+ B-cell non-Hodgkin lymphoma. J Drug Assess. 2017;6:10-7.

27. Dotan E, Cohen SJ, Starodub AN, et al. Phase I/II trial of labetuzumab govitecan (anti-CEACAM5/SN-38 antibody-drug conjugate) in patients with refractory or relapsing metastatic colorectal cancer. J Clin Oncol. 2017;35: 3338-46.

28. Socinski MA, Kaye FJ, Spigel DR, et al. Phase 1/2 study of the CD56targeting antibody-drug conjugate lorvotuzumab mertansine (IMGN901) in combination with carboplatin/etoposide in small-cell lung cancer patients with extensive-stage disease. Clin Lung Cancer. 2017;18:68-76. e62

29. Rudin CM, Pietanza MC, Bauer TM, et al. Rovalpituzumab tesirine, a DLL3targeted antibody-drug conjugate, in recurrent small-cell lung cancer: a first-in-human, first-in-class, open-label, phase 1 study. Lancet Oncol. 2017; 18:42-51.

30. Flynn MJ, Hartley JA. The emerging role of anti-CD25 directed therapies as both immune modulators and targeted agents in cancer. Br J Haematol. 2017;179:20-35

31. Horwitz SM, Fanale MA, Spira Al, et al. Interim data from the first clinical study of ADCT-301, a novel pyrrolobenzodiazapine-based antibody drug conjugate, in relapsed/refractory Hodgkin/non-Hodgkin lymphoma. Hematol Oncol. 2017;35:270-1.

32. Almhanna $K$, Wright $D$, Mercade $T M$, et al. A phase II study of antibody-drug conjugate, TAK-264 (MLN0264) in previously treated patients with advanced or metastatic pancreatic adenocarcinoma expressing guanylyl cyclase C. Investig New Drugs. 2017;35:634-41.

33. Trail PA, Dubowchik GM, Lowinger TB. Antibody drug conjugates for treatment of breast cancer: novel targets and diverse approaches in ADC design. Pharmacol Ther. 2018;181:126-42.

34. Rose AAN, Biondini M, Curiel R, Siegel PM. Targeting GPNMB with glembatumumab vedotin: current developments and future opportunities for the treatment of cancer. Pharmacol Ther. 2017:179:127-41.

35. Yardley DA, Weaver R, Melisko ME, et al. EMERGE: a randomized phase I study of the antibody-drug conjugate glembatumumab vedotin in advanced glycoprotein NMB-expressing breast cancer. J Clin Oncol. 2015;33: 1609-19.

36. Gomez-Roca CA, Boni V, Moreno V, et al. A phase I study of SAR566658, an anti CA6-antibody drug conjugate (ADC), in patients (Pts) with CA6-positive advanced solid tumors (STs) (NCT01156870). J Clin Oncol. 2016:34:2511. 
37. Forero A, Burris H III, Mita M, et al. Abstract P3-14-05: interim analysis of a phase 1 study of the antibody-drug conjugate SGN-LIV1A in patients with metastatic breast cancer. Cancer Research. 2016;76:P3-14-05-P13-14-05.

38. Sachdev JC, Maitland M, Sharma M, et al. A phase 1 study of PF-06647020, an antibody-drug conjugate (ADC) targeting protein tyrosine kinase 7 (PTK7), in patients with advanced solid tumors including platinum resistant ovarian cancer (OVCA). Ann Oncol. 2016;27:LBA35.

39. Garrido-Laguna I, Krop IE, Burris H, et al. A phase I study of PF-06647263, a novel EFNA4-ADC, in patients with metastatic triple negative breast cancer. J Clin Oncol. 2017;35:2511.

40. Kogawa T, Yonemori K, Naito Y, et al. Phase 1/2, multicenter, nonrandomized, open-label, multiple-dose first-in-human study of U3-1402 (anti-HER3 antibody drug conjugate) in subjects with HER3-positive metastatic breast cancer. J Clin Oncol. 2017;35:TPS1116.

41. Lassen UN, Ramalingam SS, Lopez JS, et al. GCT1021-01, a first-in-human, open-label, dose-escalation trial with expansion cohorts to evaluate safety of Axl-specific antibody-drug conjugate (HuMax-Axl-ADC) in patients with solid tumors (NCT02988817). J Clin Oncol. 2017;35:TPS2605.

42. Zhang C, Liu J, Zhong JF, Zhang X. Engineering CAR-T cells. Biomark Res. 2017;5:22.

43. Fesnak $A D$, June $C H$, Levine BL. Engineered T cells: the promise and challenges of cancer immunotherapy. Nat Rev Cancer. 2016;16:566-81.

44. Qin L, Zhao R, Li P. Incorporation of functional elements enhances the antitumor capacity of CAR T cells. Exp Hematol Oncol. 2017;6:28.

45. Bryan WW. Kymriah (tisagenlecleucel) approval letter. In: Drug approvals and Databases. U.S. Food and Drug Administration, Center for Drug Evaluation and Research. 2017. https://www.fda.gov/downloads/

BiologicsBloodVaccines/CellularGeneTherapyProducts/ApprovedProducts/ UCM574106.pdf. Accessed 2 September 2017.

46. Zhang $\mathrm{E}, \mathrm{Xu} \mathrm{H}$. A new insight in chimeric antigen receptor-engineered $\mathrm{T}$ cells for cancer immunotherapy. J Hematol Oncol. 2017;10:1.

47. Yu S, Li A, Liu Q, et al. Chimeric antigen receptor T cells: a novel therapy for solid tumors. J Hematol Oncol. 2017;10:78.

48. Lee DW, Gardner R, Porter DL, et al. Current concepts in the diagnosis and management of cytokine release syndrome. Blood. 2014;124:188-95.

49. Liu B, Song Y, Liu D. Clinical trials of CAR-T cells in China. J Hematol Oncol. 2017;10:166

50. van Schalkwyk MCl, Papa SE, Jeannon JP, et al. Design of a phase I clinical trial to evaluate intratumoral delivery of ErbB-targeted chimeric antigen receptor T-cells in locally advanced or recurrent head and neck cancer. Hum Gene Ther Clin Dev. 2013;24:134-42.

51. Papa S, Adami A, Metoudi M, et al. Abstract CT118: T4 immunotherapy of head and neck squamous cell carcinoma using pan-ErbB targeted CAR Tcells. Cancer Res. 2017;77:CT118.

52. Stefanski J, Brentjens R, Hollyman D, et al. CD19-targeted normal and CLL patient T cells expanded with beads can eradicate systemic tumors in vivo. Mol Ther. 2006;13:S102

53. Park JH, Rivere I, Wang X, et al. Abstract CT078: impact of disease burden and transplant on long-term survival after CD19 CAR therapy in adults with relapsed B-cell acute lymphoblastic leukemia. Cancer Res. 2017;77:CT078.

54. Locke FL, Neelapu SS, Bartlett NL, et al. Clinical and biologic covariates of outcomes in ZUMA-1: a pivotal trial of axicabtagene ciloleucel (axi-cel; KTEC19) in patients with refractory aggressive non-Hodgkin lymphoma ( $r-N H L)$. J Clin Oncol. 2017;35:7512

55. Xia L, Chen Q, Li Q, et al. Abstract CT041: the clinical study on CD19directed chimeric antigen receptor-modified T cells in patient with Richter syndrome. Cancer Res. 2017;77:CT041.

56. Turtle CJ, Hanafi L-A, Berger $C$, et al. Rate of durable complete response in $\mathrm{ALL}, \mathrm{NHL}$, and CLL after immunotherapy with optimized lymphodepletion and defined composition CD19 CAR-T cells. J Clin Oncol. 2016;34:102.

57. Fan F, Zhao W, Liu J, et al. Durable remissions with BCMA-specific chimeric antigen receptor (CAR)-modified T cells in patients with refractory/relapsed multiple myeloma. J Clin Oncol. 2017;35:LBA3001.

58. Li WP, Guo $\sqcup$, Ekaterina M, et al. Immunotherapy of hepatocellular carcinoma with T cells engineered to express glypican-3-specific chimeric antigen receptors. Mol Ther. 2015;23:S164-5.

59. Zhai B, Shi D, Gao H, et al. A phase I study of anti-GPC3 chimeric antigen receptor modified T cells (GPC3 CAR-T) in Chinese patients with refractory or relapsed GPC3+ hepatocellular carcinoma $(r / r$ GPC3+ HCC). J Clin Oncol. 2017;35:3049.
60. Wang Y, Chen M, Wu Z, et al. CD133-redirected chimeric antigen receptor engineered autologous T-cell treatment in patients with advanced and metastatic malignancies. J Clin Oncol. 2017;35:3042.

61. Cohen AD, Garfall AL, Stadtmauer EA, et al. B-cell maturation antigen (BCMA)-specific chimeric antigen receptor T cells (CART-BCMA) for multiple myeloma (MM): initial safety and efficacy from a phase I study. Blood. 2016;128:1147.

62. Berdeja JG, Lin Y, Raje NS, et al. First-in-human multicenter study of bb2121 anti-BCMA CAR T-cell therapy for relapsed/refractory multiple myeloma: updated results. J Clin Oncol. 2017;35:3010.

63. Guo B, Chen M, Han Q, et al. CD138-directed adoptive immunotherapy of chimeric antigen receptor (CAR)-modified T cells for multiple myeloma. J Cellular Immunotherapy. 2016;2:28-35.

64. Ramos CA, Savoldo B, Torrano V, et al. Clinical responses with T lymphocytes targeting malignancy-associated kappa light chains. J Clin Investig. 2016;126:2588-96.

65. Falini B, Pileri S, Pizzolo G, et al. CD30 (Ki-1) molecule: a new cytokine receptor of the tumor necrosis factor receptor superfamily as a tool for diagnosis and immunotherapy. Blood. 1995;85:1-14.

66. Ramos CA, Ballard B, Zhang H, et al. Clinical and immunological responses after CD30-specific chimeric antigen receptor-redirected lymphocytes. J Clin Invest. 2017;127:3462-71.

67. Brown CE, Alizadeh D, Starr R, et al. Regression of Glioblastoma after chimeric antigen receptor T-cell therapy. N Engl J Med. 2016;375:2561-9.

68. Soriani A, Fionda C, Ricci B, et al. Chemotherapy-elicited upregulation of NKG2D and DNAM-1 ligands as a therapeutic target in multiple myeloma. Oncoimmunology. 2013;2:e26663.

69. Klampatsa A, Haas AR, Moon EK, Albelda SM. Chimeric antigen receptor (CAR) T cell therapy for malignant pleural mesothelioma (MPM). Cancers. 2017:9:115.

70. Govers C, Sebestyen Z, Coccoris M, et al. T cell receptor gene therapy: strategies for optimizing transgenic TCR pairing. Trends Mol Med. 2010;16:77-87.

71. Mackall C, Tap WD, Glod J, et al. Open label, non-randomized, multi-cohort pilot study of genetically engineered NY-ESO-1 specific NY-ESO-1c259t in HLA-A2+ patients with synovial sarcoma (NCT01343043). J Clin Oncol. 2017; 35:3000.

72. Draper LM, Kwong MLM, Gros A, et al. Targeting of HPV-16(+) epithelial cancer cells by TCR gene engineered T cells directed against E6. Clin Cancer Res. 2015;21:4431-9.

73. Hinrichs CS, Doran SL, Stevanovic S, et al. A phase I/II clinical trial of E6 Tcell receptor gene therapy for human papillomavirus (HPV)-associated epithelial cancers. J Clin Oncol. 2017;35:3009.

74. Hong DS, Butler MO, Sullivan RJ, et al. A phase I single arm, open label clinical trial evaluating safety of MAGE-A10c796T in subjects with advanced or metastatic head and neck, melanoma, or urothelial tumors (NCT02989064). J Clin Oncol. 2017;35:TPS3098.

75. Rosenberg SA, Yang JC, Sherry RM, et al. Durable complete responses in heavily pretreated patients with metastatic melanoma using T-cell transfer immunotherapy. Clin Cancer Res. 2011;17:4550-7.

76. Goff $\mathrm{SL}$, Dudley M, Citrin DE, et al. A randomized, prospective evaluation comparing intensity of lymphodepletion prior to adoptive transfer of tumor infiltrating lymphocytes for patients with metastatic melanoma. J Clin Oncol. 2016:34:3006.

77. Noonan KA, Huff CA, Davis J, et al. Adoptive transfer of activated marrow-infiltrating lymphocytes induces measurable antitumor immunity in the bone marrow in multiple myeloma. Sci Transl Med. 2015;7:288ra278.

78. Kaufman HL, Kohlhapp FJ, Zloza A. Oncolytic viruses: a new class of immunotherapy drugs. Nat Rev Drug Discov. 2015;14:642-62.

79. Rehman H, Silk AW, Kane MP, Kaufman HL. Into the clinic: talimogene laherparepvec (T-VEC), a first-in-class intratumoral oncolytic viral therapy. J Immunother Cancer. 2016:4:53.

80. Andtbacka RHI, Curti B, Hallmeyer S, et al. Phase II CALM extension study: enhanced immune-cell infiltration within the tumour micro-environment of patients with advanced melanoma following intralesional delivery of coxsackievirus A21. Eur J Cancer. 2015;51:S677.

81. Pandha HS, Ralph C, Harrington K, et al. Keynote-200 phase 1b: a novel combination study of intravenously delivered coxsackievirus A21 and pembrolizumab in advanced cancer patients. J Clin Oncol. 2017;35:TPS3108.

82. Silk AW, Kaufman H, Gabrail N, et al. Abstract CT026: phase 1b study of intratumoral coxsackievirus A21 (CVA21) and systemic pembrolizumab in 
advanced melanoma patients: interim results of the CAPRA clinical trial. Cancer Res. 2017;77:CT026

83. Curti BD, Richards JM, Hallmeyer S, et al. Activity of a novel immunotherapy combination of intralesional coxsackievirus A21 and systemic ipilimumab in advanced melanoma patients previously treated with anti-PD1 blockade therapy. J Clin Oncol. 2017:35:3014.

84. Bernstein V, Ellard S, Dent SF, et al. Abstract CT131: a randomized (RCT) phase II study of oncolytic reovirus (pelareorep ) plus standard weekly paclitaxel (P) as therapy for metastatic breast cancer (mBC). Cancer Res. 2017:77:CT131.

85. Mahalingam D, Fountzilas C, Moseley J, et al. A phase II study of REOLYSIN(R) (pelareorep) in combination with carboplatin and paclitaxel for patients with advanced malignant melanoma. Cancer Chemother Pharmacol. 2017;79:697-703.

86. Alonso MM, García-Moure M, Gonzalez-Huarriz M, et al. Abstract CT027: oncolytic virus DNX-2401 with a short course of temozolomide for glioblastoma at first recurrence: clinical data and prognostic biomarkers. Cancer Res. 2017;77:CT027.

87. van der Burg SH, Arens R, Ossendorp F, et al. Vaccines for established cancer: overcoming the challenges posed by immune evasion. Nat Rev Cancer. 2016;16:219-33.

88. Morgensztern D, Harb W, Schalper K, et al. MA09.06 Viagenpumatucel-L bolsters response to nivolumab therapy in advanced lung adenocarcinoma: preliminary data from the DURGA trial. J Thorac Oncol. 2017;12:S394-5.

89. Zhang K, Peng Z, Huang X, et al. Phase II trial of adjuvant immunotherapy with autologous tumor-derived Gp96 vaccination in patients with gastric cancer. J Cancer. 2017;8:1826-32

90. Gray JE, Chiappori A, Williams CC, et al. Phase I/II randomized trial of GM. CD40L vaccine plus/minus CCL21 in advanced lung adenocarcinoma: Final results. J Clin Oncol. 2016;34:9037.

91. Guo C, Manjili MH, Subjeck JR, et al. Therapeutic cancer vaccines: past, present, and future. Adv Cancer Res. 2013;119:421-75.

92. Jabulowsky RA, Loquai C, Diken M, et al. Abstract CT032: a first-in-human phase I/II clinical trial assessing novel mRNA-lipoplex nanoparticles for potent cancer immunotherapy in patients with malignant melanoma. Cancer Res. 2016;76:CT032.

93. Schmitz-Winnenthal FH, Podola L, Hohmann N, et al. A phase 1 trial extension to assess immunologic efficacy and safety of prime-boost vaccination with VXM01, an oral T cell vaccine against VEGF-receptor 2, in patients with advanced pancreatic cancer. J Clin Oncol. 2016;34:3091.

94. Shore ND, Heath El, Nordquist LT, et al. A clinical trial for the safety and immunogenicity of a DNA-based immunotherapy in men with biochemically (PSA) relapsed prostate cancer. J Clin Oncol. 2017;35:e14634.

95. Teixeira L, Medioni J, Doucet L, et al. Results of a first-in-human phase I study of INVAC-1, an optimized plasmid DNA encoding an inactive form of human telomerase reverse transcriptase (hTERT), in patients with advanced solid tumors. J Clin Oncol. 2017;35:3087.

96. Singh R, Paterson Y. Listeria monocytogenes as a vector for tumorassociated antigens for cancer immunotherapy. Expert Rev Vaccines. 2006;5: $541-52$

97. Ghamande SA, Platt D, Wheatley D, et al. Phase I study evaluating highdose treatment with ADXS11-001, a Listeria monocytogenes-listeriolysin 0 ( Lm-LLO) immunotherapy, in women with cervical cancer. J Clin Oncol. 2016:34:e14580.

98. Jonker DJ, Hotte SJ, Abdul Razak AR, et al. Phase I study of oncolytic virus (OV) MG1 maraba/MAGE-A3 (MG1MA3), with and without transgenic MAGEA3 adenovirus vaccine (AdMA3) in incurable advanced/metastatic MAGEA3-expressing solid tumours: CCTG IND.214. J Clin Oncol. 2017;35:e14637.

99. Peace KM, Vreeland TJ, Clifton GT, et al. Abstract CT036: early trial results of an autologous tumor lysate, particle-loaded, dendritic cell (TLPLDC) vaccine in ovarian cancer patients. Cancer Res. 2017;77:СT036.

100. Wood LV, Roberson BD, Agarwal PK, et al. Association of autologous AdHER2 dendritic cell vaccination with antitumor activity and number of circulating tumor cells. J Clin Oncol. 2017;35:3089.

101. Pollack S, Lu H, Somaiah N, et al. Association of CMB305 or LV305-induced and baseline anti-NY-ESO-1 immunity with survival in recurrent cancer patients. J Clin Oncol. 2017;35:3090.

102. Heery CR, Palena C, McMahon S, et al. Phase I study of a poxviral TRICOM-based vaccine directed against the transcription factor brachyury. Clin Cancer Res. 2017;23:6833.

103. Sonpavde G, McMannis JD, Bai $Y$, et al. Phase I trial of antigen-targeted autologous dendritic cell-based vaccine with in vivo activation of inducible CD40 for advanced prostate cancer. Cancer Immunol Immunother. 2017:66:1345-57.

104. Espinoza-Delgado I. Cancer vaccines. Oncologist. 2002;7:20-33.

105. Oka Y, Tsuboi A, Oji Y, et al. WT1 peptide vaccine for the treatment of cancer. Curr Opin Immunol. 2008;20:211-20.

106. Nishida S, Ishikawa T, Kokura S, et al. Randomized phase II study of WT1 peptide vaccine plus gemcitabine for advanced pancreatic ductal adenocarcinoma (PDAC): clinical efficacy and immune response. J Clin Oncol. 2016;34:3085.

107. Ueda Y, Ogura M, Miyakoshi S, et al. Phase $1 / 2$ study of the WT1 peptide cancer vaccine WT4869 in patients with myelodysplastic syndrome. Cancer Sci. 2017;108:2445-53.

108. Zauderer MG, Tsao AS, Dao T, et al. A randomized phase II trial of adjuvant galinpepimut-S, WT-1 analogue peptide vaccine, after multimodality therapy for patients with malignant pleural mesothelioma. Clin Cancer Res. 2017;23:7483-9.

109. Villella JA, Wilson MK, Berinstein NL, et al. Determination of optimal dose and treatment schedule of the immunotherapeutic vaccine, DPXSurvivac, for combination immunotherapy treatment of ovarian, fallopian tube or peritoneal cancer (OC): a phase Ib study. J Clin Oncol. 2016;34:e14577.

110. Peace KM, Mittendorf EA, Perez SA, et al. Subgroup efficacy evaluation of the AE37 HER2 vaccine in breast cancer patients in the adjuvant setting. $J$ Clin Oncol. 2017:35:3088.

111. Matsui H, Hazama S, Tamada K, et al. A phase I study of novel multi-HLAbinding peptides and a new combination of immune adjuvants against solid tumors. J Clin Oncol. 2017;35:3086.

112. Yasuda T, Nishiki K, Yoshida K, et al. Cancer peptide vaccine to suppress postoperative recurrence in esophageal SCC patients with induction of antigen-specific CD8+ T cell. J Clin Oncol. 2017;35:e14635.

113. Yamaue H, Miyazawa M, Katsuda M, et al. Phase II clinical trial using novel peptide vaccine cocktail as a postoperative adjuvant treatment for surgically resected pancreatic cancer patients. J Clin Oncol. 2016;34:e14587.

114. Slingerland M, Speetjens F, Welters M, et al. A phase I study in patients with a human papillomavirus type 16 positive oropharyngeal tumor treated with second generation synthetic long peptide vaccine conjugated to a defined adjuvant. J Clin Oncol. 2016;34:TPS3113.

115. Ammi R, De Waele J, Willemen Y, et al. Poly(l:C) as cancer vaccine adjuvant: knocking on the door of medical breakthroughs. Pharmacol Ther. 2015;146: 120-31.

116. Kyi C, Sabado RL, Saenger YM, et al. In situ, therapeutic vaccination against refractory solid cancers with intratumoral poly-ICLC: a phase I study. J Clin Oncol. 2016:34:3086.

117. Marquez Rodas I, Rodriguez-Ruiz ME, Lopez-Tarruella S, et al. First-in-human clinical trial with intratumoral BO-112 in solid malignancies: a novel immunotherapy based in double-stranded RNA (dsRNA). J Clin Oncol. 2017; 35:3082.

118. Schumacher TN, Schreiber RD. Neoantigens in cancer immunotherapy. Science. 2015;348:69-74.

119. Sahin U, Derhovanessian E, Miller M, et al. Personalized RNA mutanome vaccines mobilize poly-specific therapeutic immunity against cancer. Nature. 2017;547:222-6.

120. Ott PA, Hu Z, Keskin DB, et al. An immunogenic personal neoantigen vaccine for patients with melanoma. Nature. 2017;547:217-21.

121. Kumar V, Patel S, Tcyganov E, Gabrilovich DI. The nature of myeloid-derived suppressor cells in the tumor microenvironment. Trends in Immunology. 2016;37:208-20.

122. Liguori M, Buracchi $C$, Pasqualini $F$, et al. Functional TRAlL receptors in monocytes and tumor-associated macrophages: a possible targeting pathway in the tumor microenvironment. Oncotarget. 2016;7:41662-76.

123. Forero A, Bendell JC, Kumar P, et al. First-in-human study of the antibody DR5 agonist DS-8273a in patients with advanced solid tumors. Investig New Drugs. 2017:35:298-306.

124. Lasek W, Zagozdzon R, Jakobisiak M. Interleukin 12: still a promising candidate for tumor immunotherapy? Cancer Immunol Immunother. 2014; 63:419-35.

125. Barrett JA, Cai H, Miao J, et al. IMPS-03 intratumoral regulated expression of IL-12 as a gene therapy approach to treatment of glioma. Neuro-Oncology. 2015;17:v113.

126. Chiocca EA, Yu J, Phuphanich S, et al. Expanded phase I study of intratumoral Ad-RTS-hlL-12 plus oral veledimex: tolerability and survival in recurrent glioblastoma. J Clin Oncol. 2017;35:2044. 
127. Tomala J, Kovar M. IL-2/anti-IL-2 mAb immunocomplexes: a renascence of IL-2 in cancer immunotherapy? Oncoimmunology. 2016;5:e1102829.

128. Diab A, Tannir NM, Bernatchez C, et al. A phase 1/2 study of a novel IL-2 cytokine, NKTR-214, and nivolumab in patients with select locally advanced or metastatic solid tumors. J Clin Oncol. 2017;35:e14040.

129. Bernatchez C, Haymaker CL, Hurwitz ME, et al. Effect of a novel IL-2 cytokine immune agonist (NKTR-214) on proliferating CD8+T cells and PD-1 expression on immune cells in the tumor microenvironment in patients with prior checkpoint therapy. J Clin Oncol. 2017;35:2545.

130. Pitt JM, Marabelle A, Eggermont A, et al. Targeting the tumor microenvironment: removing obstruction to anticancer immune responses and immunotherapy. Ann Oncol. 2016;27:1482-92.

131. Moon YW, Hajjar J, Hwu P, Naing A. Targeting the indoleamine 2,3dioxygenase pathway in cancer. J Immunother Cancer. 2015;3:51.

132. Siu LL, Gelmon K, Chu Q, et al. Abstract CT116: BMS-986205, an optimized indoleamine 2,3-dioxygenase 1 (IDO1) inhibitor, is well tolerated with potent pharmacodynamic (PD) activity, alone and in combination with nivolumab (nivo) in advanced cancers in a phase 1/2a trial. Cancer Res. 2017:77:CT116.

133. Zakharia Y, Drabick JJ, Khleif $S$, et al. Updates on phase $1 \mathrm{~b} / 2$ trial of the indoleamine 2,3-dioxygenase pathway (IDO) inhibitor indoximod plus checkpoint inhibitors for the treatment of unresectable stage 3 or 4 melanoma. J Clin Oncol. 2016;34:3075

134. Bahary N, Garrido-Laguna I, Cinar P, et al. Phase 2 trial of the indoleamine 2,3-dioxygenase pathway (IDO) inhibitor indoximod plus gemcitabine/nabpaclitaxel for the treatment of metastatic pancreas cancer: interim analysis. Clin Oncol. 2016;34:3020.

135. Jha GG, Gupta S, Tagawa ST, et al. A phase II randomized, double-blind study of sipuleucel-T followed by IDO pathway inhibitor, indoximod, or placebo in the treatment of patients with metastatic castration resistant prostate cancer (mCRPC). J Clin Oncol. 2017;35:3066.

136. Hamid O, Bauer TM, Spira Al, et al. Safety of epacadostat $100 \mathrm{mg}$ bid plus pembrolizumab $200 \mathrm{mg}$ Q3W in advanced solid tumors: phase 2 data from ECHO-202/KEYNOTE-037. J Clin Oncol. 2017;35:3012.

137. Perez RP, Riese MJ, Lewis KD, et al. Epacadostat plus nivolumab in patients with advanced solid tumors: preliminary phase I/II results of ECHO-204. Clin Oncol. 2017:35:3003.

138. Lu H. TLR agonists for cancer immunotherapy: tipping the balance between the immune stimulatory and inhibitory effects. Front Immunol. 2014;5:83.

139. Gupta S, Grilley-Olson J, Hong D, et al. Abstract CT091: safety and pharmacodynamic activity of MEDI9197, a TLR 7/8 agonist, administered intratumorally in subjects with solid tumors. Cancer Res. 2017;77:CT091.

140. Dredge K, Brennan T, Brown MP, et al. An open-label, multi-center phase I study of the safety and tolerability of the novel immunomodulatory agent PG545 in subjects with advanced solid tumors. J Clin Oncol. 2017:35:3083.

141. de la Torre AN, Contractor S, Castaneda I, et al. A phase I trial using local regional treatment, nonlethal irradiation, intratumoral and systemic polyinosinic-polycytidylic acid polylysine carboxymethylcellulose to treat liver cancer: in search of the abscopal effect. J Hepatocell Carcinoma. 2017; 4:111-21.

142. Ananieva E. Targeting amino acid metabolism in cancer growth and antitumor immune response. World J Biol Chem. 2015;6:281-9.

143. Papadopoulos KP, Tsai FY-C, Bauer TM, et al. CX-1158-101: a first-in-human phase 1 study of CB-1158, a small molecule inhibitor of arginase, as monotherapy and in combination with an anti-PD-1 checkpoint inhibitor in patients (pts) with solid tumors. J Clin Oncol. 2017;35:3005.

144. Zhou H, Forveille S, Sauvat A, et al. The oncolytic peptide LTX-315 triggers immunogenic cell death. Cell Death Dis. 2016;7:e2134.

145. Spicer JF, Baurain J-F, Awada A, et al. LTX-315, an oncolytic peptide, to convert immunogenically 'cold' tumors to 'hot' in patients with advanced or metastatic tumours: results from an ongoing phase I study. J Clin Oncol. 2017:35:3085

\section{Submit your next manuscript to BioMed Central and we will help you at every step:}

- We accept pre-submission inquiries

- Our selector tool helps you to find the most relevant journal

- We provide round the clock customer support

- Convenient online submission

- Thorough peer review

- Inclusion in PubMed and all major indexing services

- Maximum visibility for your research

Submit your manuscript at www.biomedcentral.com/submit

) Biomed Central 